\title{
Review Article \\ Current Trends in Nanoporous Anodized Alumina Platforms for Biosensing Applications
}

\author{
Ganesan Sriram, ${ }^{1}$ Pravin Patil, ${ }^{1}$ Mahesh P. Bhat, ${ }^{1}$ Raveendra M. Hegde, ${ }^{1}$ \\ Kanalli V. Ajeya, ${ }^{1}$ Iranna Udachyan, ${ }^{1}$ M. B. Bhavya, ${ }^{1}$ Manasa G. Gatti, ${ }^{1}$ \\ U. T. Uthappa, ${ }^{1}$ Gururaj M. Neelgund, ${ }^{2}$ Ho-Young Jung, ${ }^{3}$ Tariq Altalhi, ${ }^{4}$ \\ Madhuprasad, ${ }^{1}$ and Mahaveer D. Kurkuri ${ }^{1}$ \\ ${ }^{1}$ Centre for Nano and Material Sciences, Jain University, Jain Global Campus, Bangalore-562 112, Karnataka, India \\ ${ }^{2}$ Department of Chemistry, Prairie View A \& M University, Prairie View, TX 77446, USA \\ ${ }^{3}$ Department of Environmental Energy Engineering, Chonnam National University, Gwangju 500-757, Republic of Korea \\ ${ }^{4}$ Department of Chemistry, Faculty of Science, Taif University, Taif, Saudi Arabia
}

Correspondence should be addressed to Ho-Young Jung; jungho@chonnam.ac.kr, Madhuprasad; madhuprasad@jainuniversity.ac.in, and Mahaveer D. Kurkuri; mahaveer.kurkuri@jainuniversity.ac.in

Received 4 March 2016; Revised 24 June 2016; Accepted 11 July 2016

Academic Editor: R. Torrecillas

Copyright (C) 2016 Ganesan Sriram et al. This is an open access article distributed under the Creative Commons Attribution License, which permits unrestricted use, distribution, and reproduction in any medium, provided the original work is properly cited.

\begin{abstract}
Pristine aluminum (Al) has received great deal of attention on fabrication of nanoporous anodized alumina (NAA) with arrays of nanosized uniform pores with controllable pore sizes and lengths by the anodization process. There are many applications of NAA in the field of biosensors due to its numerous key factors such as ease of fabrication, high surface area, chemical stability and detection of biomolecules through bioconjugation of active molecules, its rapidness, and real-time monitoring. Herein, we reviewed the recent trends on the fabrication of NAA for high sensitive biosensor platforms like bare sensors, gold coated sensors, multilayer sensors, and microfluidic device supported sensors for the detection of various biomolecules. In addition, we have discussed the future prospectus about the improvement of NAA based biosensors for the detection of biomolecules.
\end{abstract}

\section{Introduction}

In recent years, metal oxide based nanomaterials are emerging in various areas such as thermal barrier [1], gas sensor [2] solar cells [3], drug delivery [4], and biomedical applications [5] due to ease of preparation and possibility in tailoring chemical and physical properties. Nanoporous oxide materials possess tremendous applications in water purification [6], biosensors [7], therapeutics [8], optical sensors [9], oxygen carrier [10], battery technology [11], and bioimaging [12]. Overall, nanoporous anodized alumina (NAA) has several advantages such as high surface area to volume ratio, thermal stability, chemical stability, electrical insulation, formation of unique pore structures, high porosity, self-organized pore structure, being nontoxic and bioactive, and biocompatibility. In addition, systematic arrangement of nanopores is of great interest, especially pores with high aspect ratios between pore diameters to pore length for various applications such as separation, drug delivery, and biosensing. Due to this, geometrical arrangements of pores with high surface area are promising candidates in biosensing for the detection of extremely rare/important biological entities.

Keller et al. have developed closely packed hexagonally arranged duplex structured NAA with pores and barrier type of oxide layer formation by anodic oxide coating through electrolytes [13]. The most popular fabrication method to synthesis NAA is electrochemical anodization, which has wide variety of applications in areas such as filtration process [14], biosensor [15, 16], drug delivery [17], corrosion resistant [18], oxygen sensor [19], catalysis [20], photocatalytic [21], cancer therapy [22], refractometric [23], interferometric [24], DNA sensor [25], bone implant [26], separation [27], and fluorescence detection [28]. The growth of porous structure depends on the ionic transport in anodic oxides under the 
influence of electric field by anodic polarization in a suitable electrolyte. The pore geometry (pore shape and diameter) depends on the processing parameters such as electrolyte solution, $\mathrm{pH}$, anodization voltage, current density, time of anodization, and temperature. However, precise pore diameter formation in the anodization process of pristine aluminum (Al) under applied electric field is completely not understood yet.

$\mathrm{Al}$ can be electrochemically anodized by two-step anodization process to get nanopores $[29,30]$. In anodization process, its surface is electrochemically oxidized in an electrolyte solution which leads to the formation of an oxide layer. The anodization can be done by applying voltage between an $\mathrm{Al}$ electrode (anode) and $\mathrm{Pb}$ and/or Pt electrode (cathode) arranged parallel to each other. The electric field is equally dispersed on its surface where pores are created on the surface of NAA [31]. The highly ordered porous structures are fabricated under low temperatures with strong acid electrolyte solutions (oxalic acid, phosphoric acid, and sulfuric acid) which is called the mild anodization process. Further, these self-ordered pore arrangements with narrow size distribution and high aspect ratio can be developed by applying different potentials, for example, at $25 \mathrm{~V}$ with sulfuric acid [32], $40 \mathrm{~V}$ with oxalic acid [30], and $195 \mathrm{~V}$ with phosphoric acid [33] leading to the formation of pores of diameters 65, 100, and $400 \mathrm{~nm}$, respectively. Despite being advantageous to form precise pores, the main disadvantage of mild anodization or self-ordered arrangement is that the rate of oxide growth is slower due to low current density and high processing time. On the other hand, hard anodization process can overcome the drawbacks of mild anodization process where higher current densities and faster processing steps are involved and the whole process is mechanically robust [34]. Recently Losic and Santos reported the fabrication of NAA by soft and hard anodization techniques for structural changes of pores and optical properties for specific sensing applications [35]. However, by using acid solutions porous structures can be created in NAA; on the other hand, by using neutral solutions barrier (nonporous) type of NAA is created [36]. Further, Lee and Park published a review on the formation of structural changes of porous and nonporous (barrier) types of NAA by anodization (Figure 1) [37].

In addition to that, NAA has been used in separation studies such as protein [38], molecular release [39], and transport [27]. This process offers self-organized growth of nanopores from top to bottom which provides highly ordered NAA structure. The fabrication of nanopores in NAA of different structures such as funnel [40], multilayered [41], hierarchical [42], and three-dimensional [43] has also been reported. Recently, Santos et al. developed NAA with inverted funnel structure; this type of multistratified structures of cylindrical nanopores varies in pore diameter from top to bottom [44]. The NAA can also be fabricated in wide variety of nanostructures such as nanotubes, nanofibers [45], and nanodots [46].

Owing to the abovementioned importance and wide range of NAA applications herein, we are reviewing fabrication and applications of NAA in biosensing arena. In this article, the most recent and current research on NAA

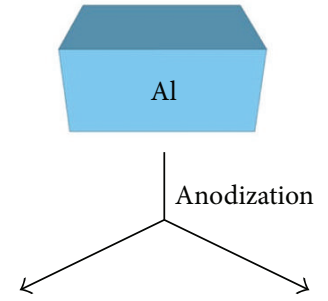

$\begin{array}{ll}\text { (a) Barrier, type NAA } & \text { (b) Porous, type NAA }\end{array}$

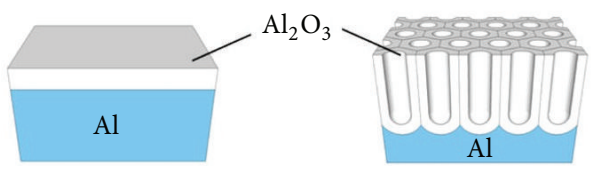

FIGURE 1: Specific diagram of formation of porous and nonporous NAA by Al through anodization process [37].

and gold coated NAA (Au-NAA) with their key role in biosensing applications have been covered. In particular, our focus is on fabrication of NAA by anodization process and their specific applications in biosensing with detailed description on fabrication conditions, working principles, and mechanism of biosensing. Finally, the future prospectus of NAA in the area of biosensing has been discussed.

\section{Fabrication of Nanoporous Anodized Alumina (NAA)}

In general, NAA can be developed by two-step anodization method (Figure 2) [47, 48]. As discussed in Introduction, by varying parameters like applied current, concentration of acid solutions, anodization time, and temperature, one can tailor the NAA properties. For example, Yin et al. used same method [63]; at first the Al film was electrochemically polished in a mixture of ethanol (EtOH) and $\mathrm{HClO}_{4}$. In the next step, $\mathrm{Al}$ was anodized with $0.3 \mathrm{M}$ oxalic acid at $10^{\circ} \mathrm{C}$ and at $40 \mathrm{~V}$ for few hours to obtain nanoporous structures of NAA. Further, second anodization was carried out in the same way to remove the barrier layer which is created at the bottom of nanopores by using $0.5 \mathrm{M}$ phosphoric acid or by using dry etchants like chlorine-based gases. In this method highly ordered nanopore arrays in thin alumina film on silicon substrate were obtained.

Similarly, Lo and Budiman fabricated NAA by using impure $\mathrm{Al}$ foils which can be used in large scale applications whereas the highly pure foils have limited applications in nanoelectrophotonics. The impure $\mathrm{Al}$ foil was taken as anode and copper as cathode [64]; further, $5 \times 5 \mathrm{~mm}$ pieces of impure $\mathrm{Al}$ foil were mounted on the glass slides and these were then anodized in $0.3 \mathrm{M}$ oxalic acid for about $18 \mathrm{~h}$ at constant voltage of $40 \mathrm{~V}$. The oxidized layer was etched using $0.5 \mathrm{M}$ phosphoric acid at $80^{\circ} \mathrm{C}$ for $1 \mathrm{~h}$. The same material was anodized by using above conditions for $15 \mathrm{~min}$ to develop nanopores throughout the surface. Thus, obtained samples were removed and floated over saturated $\mathrm{HgCl}_{2}$ and further treated with $\mathrm{H}_{3} \mathrm{PO}_{4}$ to remove the deposited barriers to get NAA. To compare, ultrapure $\mathrm{Al}$ foils were used and it was found that all the properties such as growth rate and chemical 


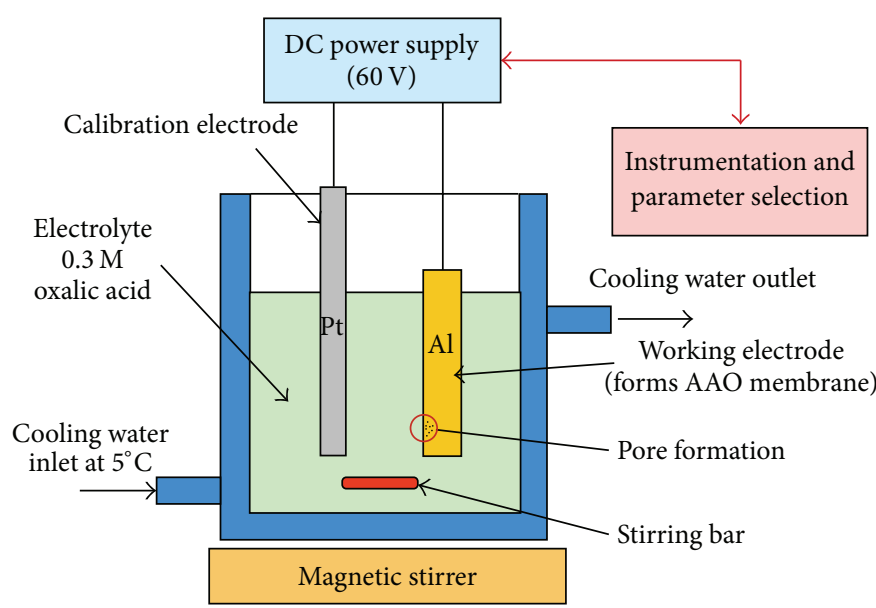

(a)

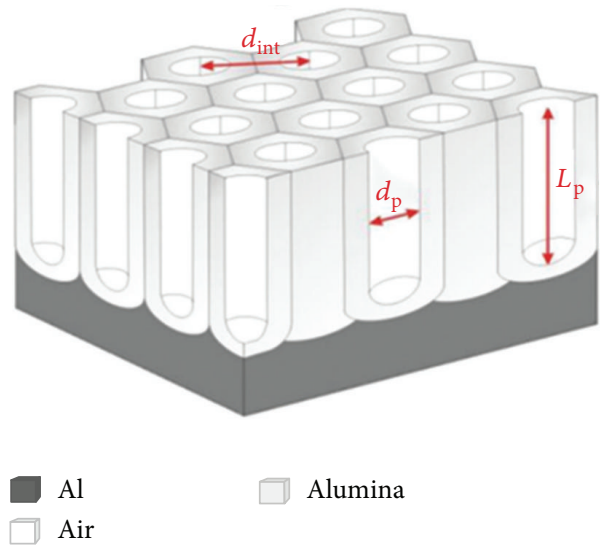

(b)

Figure 2: (a) Experimental set-up for fabrication of NAA by two-step anodization process and (b) the geometric structure of NAA ( $d_{\text {int }}$ : interpore distance, $d_{\mathrm{p}}$ : pore diameter, and $L_{\mathrm{p}}$ : pore length) $[47,48]$.

composition were similar with that of NAA produced from impure $\mathrm{Al}$ foils except the pore ordering (morphology). However, the pore consistency and order can be controlled by electropolishing the impure $\mathrm{Al}$ foil.

Nagaura et al. proposed a multistep anodization and leaching process to get three dimensional structured films [65], in which $\mathrm{Al}$ foils were used as working electrode in anodization process and carbon electrode as counter electrode. Each anodization step was followed by dipping the substrate into phosphoric acid for pore widening, which was called leaching process. The anodization was conducted in phosphoric acid solution at $10^{\circ} \mathrm{C}$ with stirring and varying current to get multistep structures which is followed by leaching process after every anodization step with different time interval of dipping.

Similarly, Hussain et al. fabricated uniform NAA to fabricate tungsten trioxide $\left(\mathrm{WO}_{3}\right)$ nanowires [66]. In this process, $\mathrm{Al}$ foils were cleaned with acetone to remove the dirt and greases which were further subjected to annealing at $500^{\circ} \mathrm{C}$ for $5 \mathrm{~h}$ at heating rate of $10^{\circ} \mathrm{C} / \mathrm{min}$. Further, the oxides were removed by dilute oxalic acid and rinsed with deionized water. In next step, electrically polished foils were kept in a mixture of EtOH and perchloric acid at a voltage of $12 \mathrm{~V}$ for $3 \mathrm{~min}$ at $100^{\circ} \mathrm{C}$. Further, two-electrode cell system was used to fabricate NAA using graphite as counter electrode. Anodization was carried out at $40 \mathrm{~V}$ for $120 \mathrm{~min}$ at $50^{\circ} \mathrm{C}$ in $0.3 \mathrm{M}$ $\mathrm{H}_{2} \mathrm{C}_{2} \mathrm{O}_{4}$ electrolyte. The oxide layer of NAA substrate was removed by washing with $\mathrm{H}_{2} \mathrm{Cr}_{2} \mathrm{O}_{4}$ and $\mathrm{H}_{3} \mathrm{PO}_{4}$ for $30 \mathrm{~min}$. Further, it was again anodized at a voltage of $40 \mathrm{~V}$ for $120 \mathrm{~min}$ at $50^{\circ} \mathrm{C}$ in $0.3 \mathrm{M} \mathrm{H}_{2} \mathrm{C}_{2} \mathrm{O}_{4}$ acid. After this process, NAA was immersed in $\mathrm{H}_{3} \mathrm{PO}_{4}$ for 90 min to increase the size of pore width. The fabricated NAA has been used as a template to fabricate $\mathrm{WO}_{3}$ nanowires by electroless deposition bath method. NAA was sonicated under the mixture of $\mathrm{SnCl}_{2}$ and $\mathrm{HCl}$ in the $\mathrm{wt} \%$ of 0.3 and 2.5, respectively. However, in this mixture, dissolution of the template was due to prolonged dipping process. On the other hand, NAA was sonicated under the mixture of $\mathrm{PdCl}_{2}$ and $\mathrm{HCl}$ used in the $\mathrm{wt} \%$ of 0.1 and 1, respectively, for $3 \mathrm{~min}$ which is followed by dipping the template in deionized water. The deposition bath was used to form nanowires by NAA template in which sodium tungstate, hydrazine hydrate, sodium potassium tartrate, and acetic acid were used as a composition. With this, $\mathrm{WO}_{3}$ nanowires were formed in the pores of NAA.

For the formation of unidirectional NAA structures $\mathrm{Na}$ et al. [67] used pure $1050 \mathrm{Al}$ sheets after pretreatment to clean the surface before the anodization process. $\mathrm{Al}$ samples were anodized in dilute $\mathrm{H}_{2} \mathrm{SO}_{4}$ electrolyte solution using Pt cathode at a current density of $3 \mathrm{~A} / \mathrm{dm}^{2}$. In the next step, anodization was carried out at different electrolyte temperatures such as 2,10 , and $15^{\circ} \mathrm{C}$ for 5 to $6 \mathrm{~h}$. After this process, acid resistant tape was removed by acetone washing to form porous structures on surface. The thickness and average diameter of unidirectional nanopore were found to be $230-240 \mu \mathrm{m}$ and $6-24 \mathrm{~nm}$, respectively.

In another work, the synthesis of carbon nanotubes in NAA film by $\mathrm{H}_{2} \mathrm{SO}_{4}$ or $\mathrm{H}_{2} \mathrm{C}_{2} \mathrm{O}_{4}$ acid was achieved by Sui et al. [45]. Polished Al plate was anodized at different anodic voltages in $0.16 \mathrm{M} \mathrm{H}_{2} \mathrm{C}_{2} \mathrm{O}_{4}$ or $1.2 \mathrm{M} \mathrm{H}_{2} \mathrm{SO}_{4}$ solution for $25 \mathrm{~min}$ by rigorous stirring at $15^{\circ} \mathrm{C}$ to get porous NAA template, which was then cleaned and kept for boiling $(15 \mathrm{~min})$. Pairs of alumina templates with or without boiling in water were put into tubular stove with gas flow mixture of $\mathrm{N}_{2} \mathrm{C}_{2} \mathrm{H}_{2}$ at the rate of $100 \mathrm{~mL} / \mathrm{min}$ at a particular temperature for $2 \mathrm{~h}$. Further, $\mathrm{Al}$ was dissolved in $10 \% \mathrm{NaOH}$ solution to release nanotubes and nanofibers. Immediately, these were thoroughly washed and dispersed in pure EtOH solution by sonication method to obtain pure nanopore structures in the form of carbon nanotubes on NAA.

Furthermore, Tsuchiya et al. developed self-organized NAA in neutral electrolytes [68]. Al foils $(0.5 \mathrm{~mm})$ were degreased by sonicating in acetone, isopropanol, and methanol followed by rinsing in deionized water and dried under nitrogen atmosphere. Anodization was done in 
a conventional cell with three electrodes; Pt as counter electrode and Haber-Luggin capillary electrode with $\mathrm{Ag} / \mathrm{AgCl}$ were as reference electrode. The samples were assembled in electrochemical cell by pressing against an $\mathrm{O}$-ring. The electrolytes such as $1 \mathrm{M}\left(\mathrm{NH}_{4}\right)_{2} \mathrm{SO}_{4}$ solution with fluoride ions and deionized water were used in this experiment. Anodization was done at high voltage potentiostat which consists of potential from open circuit potential to certain fixed potential. Further, the samples were rinsed in deionized water and dried in nitrogen stream. The pore diameter of NAA was obtained at different potentials in acid solutions ranging from 65 to $500 \mathrm{~nm}$.

Fabrication of NAA with ideally ordered channel arrangements was reported by Asoh et al. [69]. In this, the electropolished master mold was placed on pure $\mathrm{Al}$ sheet below $10^{\circ} \mathrm{C}$ for about $4 \mathrm{~min}$ in a mixed solution of $\mathrm{HClO}_{4}$ and $\mathrm{C}_{2} \mathrm{H}_{5} \mathrm{OH}$. An array of concaves on $\mathrm{Al}$ surface was formed. Further, anodization of $\mathrm{Al}$ was done by $0.3 \mathrm{M} \mathrm{H}_{2} \mathrm{SO}_{4}$ and/or $\mathrm{H}_{2} \mathrm{C}_{2} \mathrm{O}_{4}$ under constant voltage. Later, anodic oxide film was removed from $\mathrm{Al}$ substrate in saturated $\mathrm{HgCl}_{2}$ solution. The selective removal of barrier layer (down part of oxide layer) and pore widening were done by etching the NAA membrane in $5 \mathrm{wt} \%$ $\mathrm{H}_{3} \mathrm{PO}_{4}$ at $30^{\circ} \mathrm{C}$. This treatment facilitates better observation of the holes at bottom oxide layer, where uniform diameter of channels $(40 \mathrm{~nm})$ and depth of the nanopore $(1 \mu \mathrm{m})$ were formed.

On the other hand, Zhang et al. fabricated NAA membrane using low potential anodizing process [70]. Highly pure $\mathrm{Al}$ foils were cut into small pieces and annealed in a furnace in the presence of air at $600^{\circ} \mathrm{C}$. Mixture of acids $\left(\mathrm{H}_{3} \mathrm{PO}_{4}: \mathrm{HNO}_{3}: \mathrm{H}_{2} \mathrm{O}=8: 1: 1\right)$ was taken and $\mathrm{Al}$ plates were polished for $30 \mathrm{~s}$ at $95^{\circ} \mathrm{C}$ followed by electrochemical polishing in perchloric acid and $\mathrm{EtOH}$ (1:4 ratio). Anodization was done in $\mathrm{H}_{2} \mathrm{SO}_{4}$ aqueous solution under constant potential of $18 \mathrm{~V}$. The Al plate was fixed in acid solution which was separated from cathode of mechanically polished copper plate. All experiments were conducted in $3^{\circ} \mathrm{C}$ cold water using ice bath. The oxide layer was produced at first anodization step and was removed by submerging the Al plate in chromic acid solution. Second anodization was conducted under same conditions by shortening the duration to $3 \mathrm{~h}$. The NAA were obtained with pore diameters ranging from 6.8 to $19.3 \mathrm{~nm}$ at potential of 6 to $18 \mathrm{~V}$.

Wang et al. fabricated NAA nanotubes using pulse anodization; the NAA nanotubes were fabricated by modified anodization process, that is, pulse anodization [71]. Al plate was sonicated in $\mathrm{EtOH}$, ultrapure water, and then electropolished in a mixture of EtOH and $\mathrm{HClO}_{4} 4: 1(\mathrm{v}: \mathrm{v})$ at $20^{\circ} \mathrm{C}$ and $5^{\circ} \mathrm{C}$ for $3 \mathrm{~min}$. The first anodization was done in an electrolyte solution of $0.3 \mathrm{M} \mathrm{H}_{2} \mathrm{SO}_{4}$ at $25 \mathrm{~V}$ and $6^{\circ} \mathrm{C}$. This consists of mild anodization and hard anodization pulses in $0.3 \mathrm{M} \mathrm{H}_{2} \mathrm{SO}_{4}$ electrolyte and $\mathrm{EtOH}$ of concentration ranging from 0 to $25 \%$. Wet chemical etchants were used to remove remaining $\mathrm{Al}$ substrates in $0.2 \mathrm{M} \mathrm{CuCl}_{2}$ and $6.1 \mathrm{M} \mathrm{HCl}$ solution. Through pulse anodization, the pore length and diameters of NAA were found to be 500 and $90 \mathrm{~nm}$, respectively.

Friedman et al. prepared series of highly porous and pure NAA [72]. A flat cell was used with a Pt mesh counter electrode, and the $\mathrm{Al}$ was exposed to electrolyte at $0.25 \pi \mathrm{cm}^{2}$.
At first anodization step, the NAA was anodized for $2 \mathrm{~h}$ and kept in $5 \% \mathrm{H}_{3} \mathrm{PO}_{4} / 5 \%$ chromic acid solution for overnight which is followed by etching to provide a barrier layer, thus resulting in the formation of small porous structures on the surface of Al. Further, it was reanodized at the same condition as in the first step but with shorter time interval. So, the above methods emphasize the fabrication of different types of NAA with different pore diameters and pore lengths for the detection of immobilized biomolecules on their surface. In addition, these key parameters enhance the optical properties and rapid sensitivity in the detection process of biomolecules. Consequently, the structural parameter and fabrication conditions of the NAA become crucial platform for biosensing application.

\section{Applications of NAA in Biosensing}

Recently, NAA based materials have attracted considerable attention in biosensing due to possibilities like immobilization of target molecules on nanopores, optical sensing enhancement by coating Au layer on NAA surfaces, low concentration detection, biocompatibility with living cells due to Au coating, portability, and so forth. The recent reviews on NAA based biosensing date back to 2014 [7376]; thus, there is a huge scope for the current review article which covers latest research works and current trends in this particular field. Owing to this, it could help the readers to find recent developments and current trends in NAA based biosensing applications. The following applications are categorized based on the types of transducer for the detection of biomolecules.

\section{NAA Biosensor}

4.1. Detection of Glucose. The barcode system enhances the optical biosensing effect of NAA for oxazine dye and glucose using photoluminescence (PL) spectroscopy [77]. Enhancement in the PL barcode system for optical biosensor was observed for NAA structure with wide pore diameter $(30-71 \mathrm{~nm})$ and controlled pore length $(5-12.4 \mu \mathrm{m})$. The barcodes were produced with respect to different wavelength of PL spectra. The wide pore diameter and controlled pore lengths were obtained by wet chemical etching and various anodization processing times. It was found that PL barcode system depends on the pore diameter and lengths. In a control experiment, no PL spectrum was observed for the pore length larger than $25 \mu \mathrm{m}$ with $5 \mathrm{~h}$ anodization time. In this study pore lengths, such as 5,8 , and $12.4 \mu \mathrm{m}$, resulted in better PL intensities. Intensity of PL spectra was increased with wide pore diameter, due to decrease in surface thickness of substrate. However, PL intensity has been decreased while increasing the etching time for longer pore diameters. Further enhancement in PL intensity for larger pore diameter was exhibiting better sensing effect for oxazine dyes and glucose biosensors.

Furthermore, Santos et al. studied the effect of pore length and diameter of NAA for biosensing applications [78]. The study showed effect of pore diameter and pore length with respect to particular reflection in NAA. Samples 


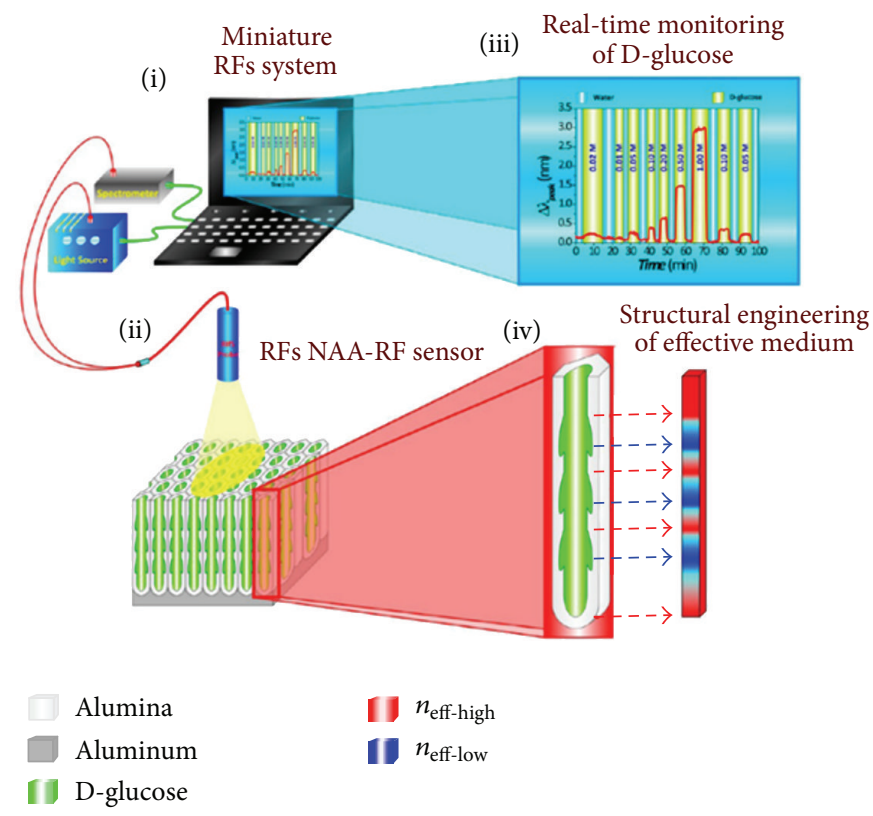

(a)

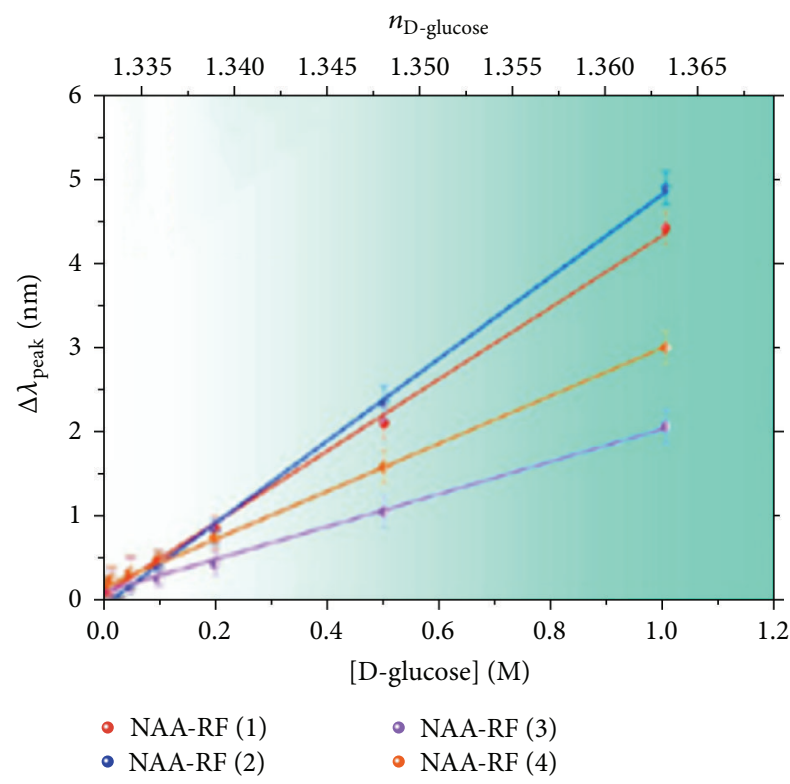

(b)

FIgURE 3: (a) Schematic diagram of sensing principle of D-glucose by NAA-RFs using reflection spectroscopy: (i) set-up of reflection spectroscopy combined with light source, spectrometer, and optical probe, (ii) optical sensing platform on NAA-RFs, (iii) real-time monitoring of D-glucose by NAA-RFs system, and (iv) structural nanopore geometry of effective medium; (b) sensing plots at different concentration of D-glucose [15].

with different pore diameters with same pore lengths were considered with same geometrical characteristics. The wider pore showed greater reflectivity due to bigger pore diameter and larger pore gives lower reflectivity due to large pore length. The reflectivity studies have been used to predict the biosensing mechanism of NAA. Furthermore, Santos et al. reported the comparision of biosensor material for the detetction of D-glucose and L-cysteine using PL and reflectrometric interference spectroscopy (RIfS) [79]. NAA substrate was fabricated by two-step anodization process with pore length and pore diamters of 1.8 to $12.7 \mu \mathrm{m}$ and 29 to $74 \mathrm{~nm}$, respectively. The detetcion of D-glucose and Lcysteine under nonspecific and specific adsorption conditions by NAA was carried out using PL and RIfS systems. However, the better sensitivity for both the biomolecules was observed for NAA by PL due to their photoactivity compared to RIfS. Sensing of glucose and L-cysteine was achieved at low concentration by NAA, that is, $0.01 \mathrm{M}$ and $0.005 \mathrm{M}$, respectively, using PL.

In another work, Kumeria et al. developed NAA rugate filters (NAA-RFs) for real-time detection of D-glucose by reflection spectroscopy with flow cell [15]. Reflection spectroscopy is one of the optical instruments with various benefits such as small size, being portable, and low cost, compared to other optical instruments. Four different types of NAA rugates, that is, NAA-RF 1, NAA-RF 2, NAA-RF 3 , and NAA-RF 4 , were fabricated. The detection of Dglucose in flow cell was observed by change in refractive index (RI) of NAA rugate filter with respect to adsorption of Dglucose into nanopores. The RI was measured initially by using distilled water which was sent into flow cell followed by D-glucose solution. Further, this D-glucose was immobilized on NAA-RF 1 where RI has been increased for D-glucose and decreased for distilled water. Similar procedure was applied for other three NAA rugate filters. However, better sensitivity was found for NAA-RF 2 compared to other three NAA-RFs materials for D-glucose. The schematic diagram explains the optical system for detection of D-glucose by combining NAARFs and reflection spectroscopy and sensing plot for different concentration of $\mathrm{D}$-glucose with respect to change in effective RI of NAA-RFs using reflection spectroscopy (Figure 3).

4.2. Detection of Binding of Antibody and Antigen. Takmakov et al. fabricated NAA for the detection of streptavidin through covalently bonded biotin on sensor surface using fluorescence microscopy [80]. By using aminosilane-succinimide chemistry [81], the biotin molecules were immobilized covalently on NAA surface. Fluorescence microscopy was used to detect streptavidin molecules on biotin bonded sensor surface. The fabrication process and sensing mechanism are explained as follows: Al film was coated on glass substrate through mask by ion beam coater with film thickness of around $600 \mathrm{~nm}$. The anodized $\mathrm{Al}$ film showed pore diameter and thickness of $20 \mathrm{~nm}$ and $60 \mu \mathrm{m}$, respectively. The biotin molecules were immobilized on sensor surface by NAA which were rinsed in 3-aminopropyltriethoxysilane (APTES) and later washed with acetone followed by drying in oven. Biotin layer was formed on sensor surface by mixing with dimethyl sulfoxide (DMSO) solution of biotin $\mathrm{N}$-hydroxysuccinimide ester and acetone. The covalently 
bonded biotins were analyzed on surface by FTIR. Further, biotinylated substrate was exposed to streptavidin modified Alexa Fluor 488 dye solution for $5 \mathrm{~min}$. The unbound streptavidin on biotinylated substrate was removed by further washing with phosphate-buffered saline (PBS) solutions. The streptavidin bounded biotin NAA substrate was immersed into water and then analyzed by fluorescence microscope to confirm the binding of biomolecules. The streptavidin molecules were exposed to both materials in which the fluorescence contrast was high for NAA compared to Al film due to high diffusion of streptavidin into porous structured NAA. The sensing of streptavidin was carried out by using fluorescence microscopy where significant increase in fluorescent intensity was observed for NAA (black line) due to covalent bonding of streptavidin-biotin on sensor surface and moreover biomolecular diffusion process was higher in porous surface. In contrast, Al film exhibited very low intensity peaks due to improper binding of biomolecules and was having less diffusion due to nonporous structure of bare metal film.

Fu et al. developed NAA based biosensor for the detection of antigen-antibody detection using SERS method [82]. NAA was developed by the process of $\mathrm{Al}$ thin film coated on glass substrate with thickness of $380 \mathrm{~nm}$ by thermal evaporation technique and followed by anodization process. Further, silver $(\mathrm{Ag})$ nanoparticles were coated on the surface of NAA to enhance the RI of medium. The immobilized antigen-antibody was obtained on modified NAA substrate by washing with APTES solution followed by GTA solution for the formation of amino and aldehyde groups, respectively. The detection of antigen-antibody recognition on NAA was observed by wave guide assisted SERS system. The obtained human IgG (antigen) was covalently bound with NAA surface followed by FITC-anti-IgG (antibody) which were allowed to immobilize with human IgG modified NAA surface. The immobilization of molecules was analyzed by change in angle dependent reflectivity on NAA surface. The angle dependent reflectivity studies were obtained by processing bare NAA substrate at angle $44.74^{\circ}$, human IgG modified NAA surface has angle of $45.04^{\circ}$, and at the end of FITCanti IgG modified NAA the angle was $44.42^{\circ}$. The above results show that the angle dependent reflectivity increases with respect to excess binding of molecules on NAA surface. The detection of binding molecules on NAA surface was analyzed by enhanced SERS signals through Ag nanoparticles accumulation.

Kant et al. developed biosensing platform by utilizing the pore dimension of NAA to detect the immobilization of streptavidin (antibody) with biotin (antigen) by electrochemical impedance spectroscopy [49]. NAA has been fabricated with $25-65 \mathrm{~nm}$ and $4-18 \mu \mathrm{m}$ pore size and lengths, respectively. In addition, nanopore arrays were patterned on back side of substrate by photolithographic process. The streptavidin biomolecules were bound on nanopore array structure of NAA surface by soaking it in mixture of streptavidin with PBS solution. The different concentration solutions of biotin molecules were allowed to bind on streptavidin functionalized substrate. However, $0.2 \mu \mathrm{M}$ concentration of biotin molecules bound with streptavidin showed better sensing performance due to decrease in pore resistance on substrates and increase in pore diameter from 25 to $65 \mathrm{~nm}$. It has been found that sensitivity has not only depended on pore dimensions where size of biomolecules may also influence the enhancement of sensing performance. The size of immobilized biomolecule of streptavidin was about 4$5 \mathrm{~nm}$ and the size increased to $6-8 \mathrm{~nm}$ due to binding with biotin molecule which resulted in better performance. The electrochemical impedance spectroscopy was performed by four electrode systems to characterize the NAA biosensor. The electrochemically impedance biosensor performed by change in surface charge of NAA binding to both biomolecule and pore resistance of substrate. Two gold foils as working and counter electrodes with $\mathrm{Ag} / \mathrm{AgCl}$ were used as a reference electrode. The NAA substrate was sandwiched between two cells and contacted to reference electrode from each side of cells. The working and counter electrodes were placed at the end of two cells. An equivalent circuit was used to balance the resistance and capacitance of the NAA to analyze the streptavidin bonded biotin on NAA. The sensitivity of NAA has been enhanced by decreasing pore resistance with respect to change in conductivity of sensor surface. The larger pores with longer pore length are not suitable for the detection of biomolecules. Thus, the pore resistance increased when the pore length was increased from 4.5 to $18 \mu \mathrm{m}$. The pore resistance depends on the pore length and it was found that no response/sensitivity was observed for the pore length above $10 \mu \mathrm{M}$. The detection limit was observed using different solutions of biotin in the range of 0.2 to $10 \mu \mathrm{M}$ by binding with streptavidin on NAA substrate, but in the concentration of $0.2 \mu \mathrm{M}$ it showed better sensitivity for the binding of biotin and streptavidin molecules on NAA substrates. The fabrication and formation of nanopore arrays on NAA surface by lithographic technique and graphs are shown in Figure 4. The graph represents the pore resistance dependent on different concentration of biotin solution with respect to different pore diameters and pore lengths obtained by NAA biosensor.

4.3. Detection of Protein. Alvarez et al. reported real-time detection of binding protein-A with rabbit, anti-sheep, and sheep immunoglobulin on the surface of NAA with respect to change in optical thickness using fluorescence microscopy [50]. The fabricated label-free NAA biosensor has structural dimensions of $60 \mathrm{~nm}$ and $6 \mu \mathrm{m}$ in diameter and thickness, respectively. The sensor surface modification by protein-A has been tested for interaction with rabbit, sheep, rabbit, anti-sheep and chicken's immunoglobulin. The binding interactions were carried out in PBS solution with $0.1 \mathrm{mg} / \mathrm{mL}$ biomolecules concentration for the biosensing applications. Schematic diagram in Figure 5 represents the protein-A modified sensor surface and step by step binding mechanism of rabbit anti-sheep and sheep's immunoglobulin biomolecules with protein-A. The sensitivity has been measured in terms of time versus change in optical thickness (OT) of sensor surface modified protein-A with rabbit, rabbit anti-sheep, sheep, and chicken immunoglobulin. Optical thickness has been rapidly increased with respect to time on NAA sensor surface for protein-A interacting with rabbit anti-sheep and 


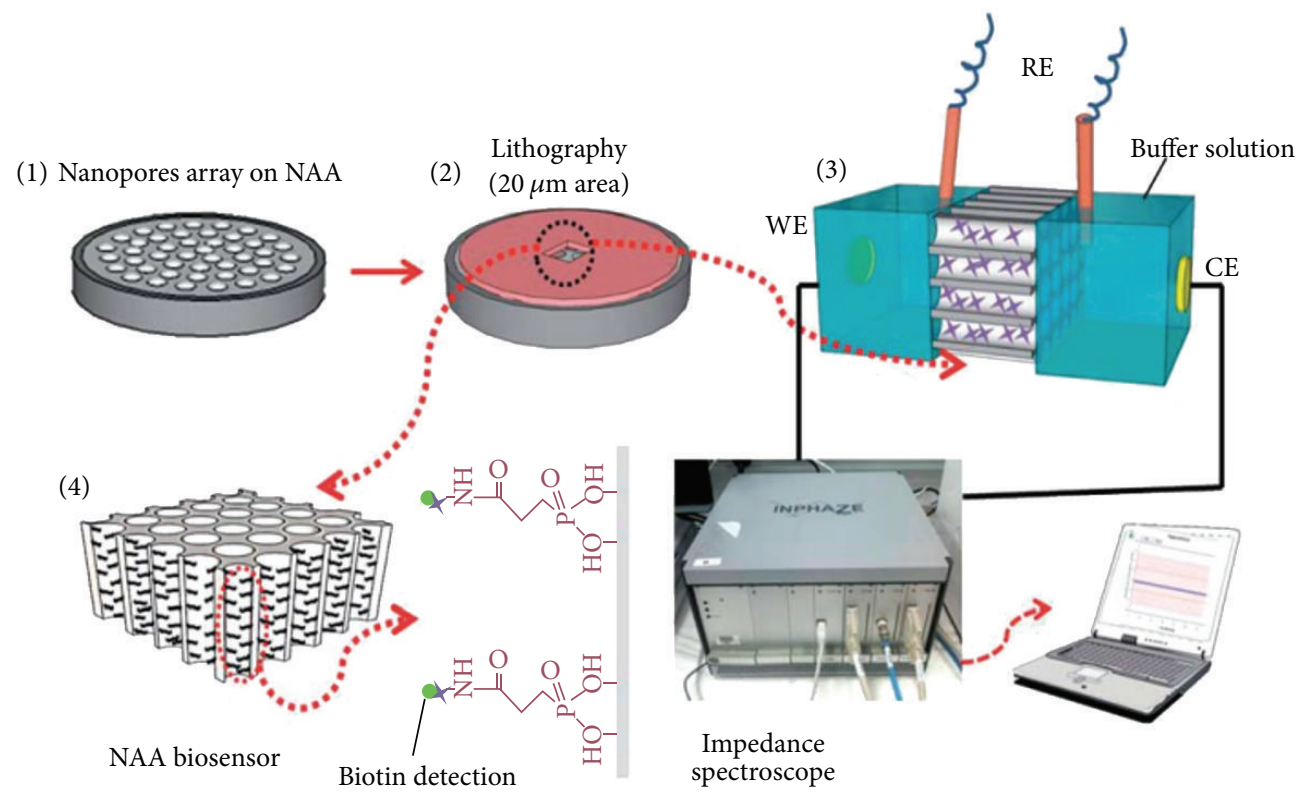

(a)

(1)

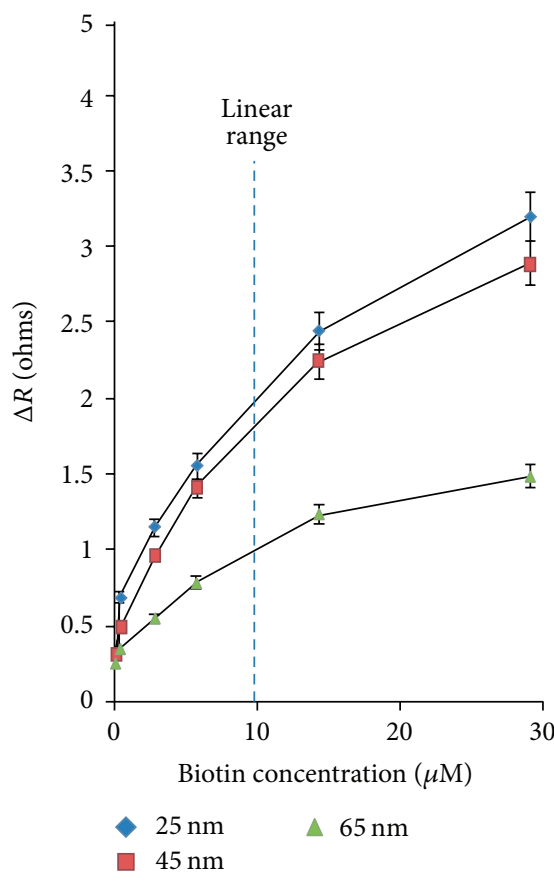

(2)

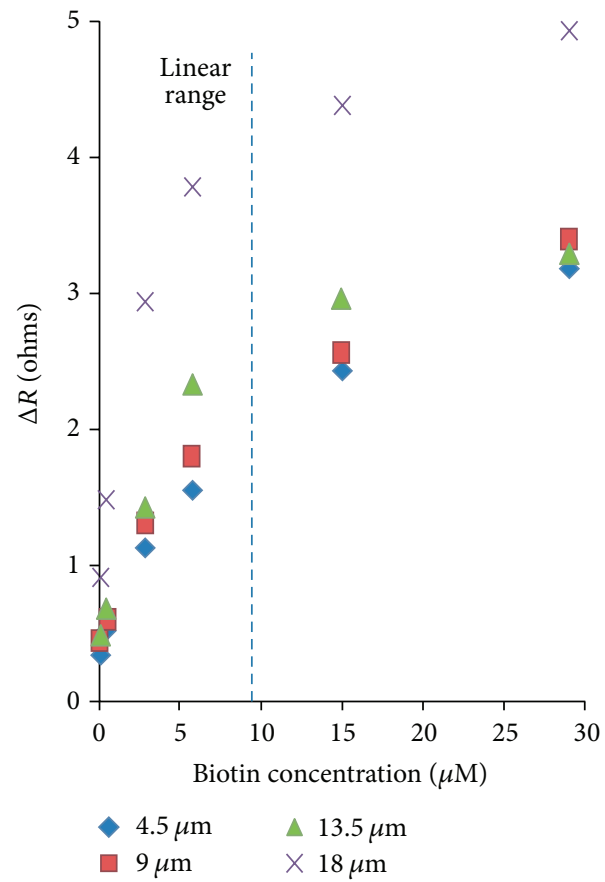

(b)

FIGURE 4: (a) Schematic representation showing fabrication process involved in the formation of NAA biosensor along with detection. (b) The graph representing changes in pore resistance with different biotin concentration and graph showing linearity between actual pore sizes with different biotin concentrations [49].

sheep immunoglobulin biomolecules. However, no binding affinity was observed for rabbit and chicken immunoglobulin biomolecules (dark base lines) due to weak interaction with sensor surface.

In addition to that, Lau et al. fabricated NAA for the detection of bovine serum albumin (BSA) through pore widening using optical waveguide spectroscopy [83]. The immobilized BSA on NAA surface was observed for reflectivity to confirm the detection. High sensing performance was attained through the pore widening on NAA surface. The reflectivity increased with respect to increase in the sensitivity of material. The enhancement in sensing performance 


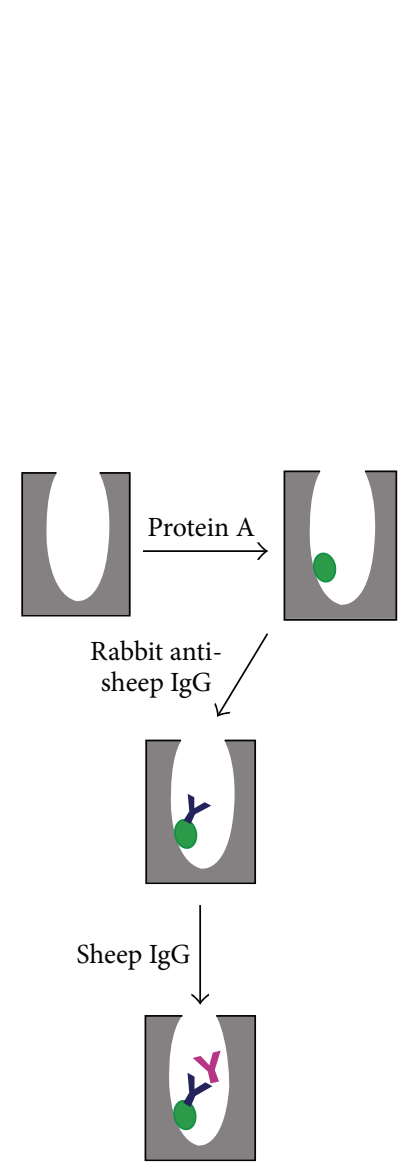

(a)
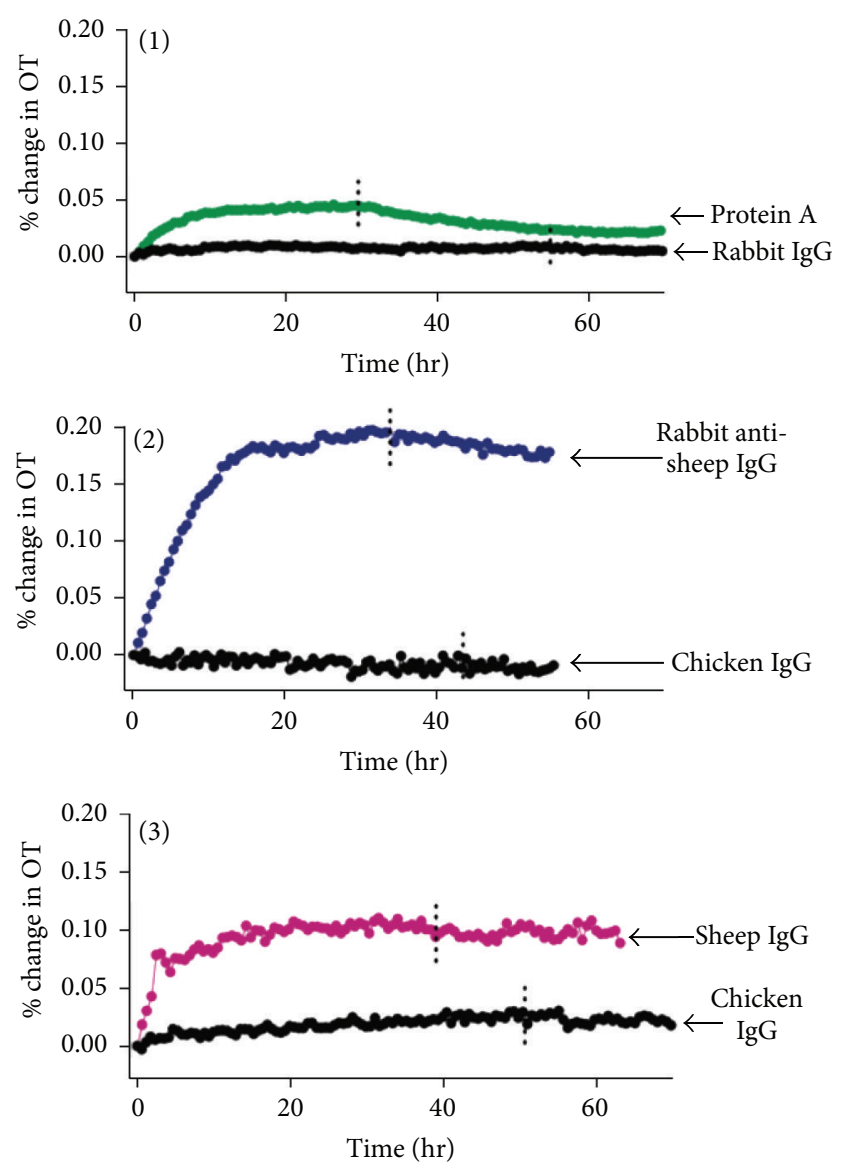

(b)

Figure 5: (a) The schematic diagram of binding affinity of biomolecules on NAA surface and (b) the time dependent change in optical thickness (OT) graphs showing the binding interaction of protein-A, rabbit, rabbit anti-sheep, sheep, and chicken immunoglobulin [50].

was obtained by processing film with uniform porous structure.

4.4. Detection of DNA. Pan and Rothberg developed immobilization of single stranded DNA (ssDNA) oligonucleotides on NAA and the fabricated materials were functionalized as a reflective biosensor to detect complementary DNA at area of $1 \mathrm{nmol} / \mathrm{cm}^{2}$ [84]. The fabricated structures of NAA were of $6 \mu \mathrm{m}$ and $40 \mathrm{~nm}$ in thickness and diameter, respectively. The fabricated sensor material has very high surface area which could easily accommodate DNA oligonucleotides on NAA surfaces. The detection of biomolecules was based on change in reflectivity on the surface of sensor material.

Takmakov et al. studied the ionic conductance via shrunk porous alumina and ssDNA biosensing by using porous material [85]. The NAA substrate was fabricated with the structural dimensions of $60 \mathrm{~nm}$ and $10 \mu \mathrm{m}$ pore diameter and depths, respectively. Further, NAA pore diameters were shrunk by hydrothermal treatment. The process involves three steps, formation of shrunk porous structures on sensor material by anodization, the reduction in the size of porous structures by hydrothermal treatment, and the detection of DNA oligomers by porous materials. The
ssDNA biomolecules were immobilized on sensor surface by aminosilane-glutaraldehyde chemistry [86]. The immobilization of ssDNA inside the sensor material decreases the pore resistance due to increase in the surface charge density of sensor material with negatively charged ssDNA. The immobilized ssDNA was hybridized with double stranded DNA (dsDNA) to increase the pore resistance. The dsDNA was denatured using urea which liberates complementary DNA strands to recover the initial pore resistance of immobilized ssDNA. The DNA detection has been achieved by the conduction variation with or without hybridization of DNA in internal nanopores of sensor material through electrical impedance studies.

Hotta et al. developed the multilayer structure polyelectrolytes coated NAA surface for the detection of DNA (single stranded probe DNA) and hybridized DNA using infrared reflection adsorption spectroscopy (IRRAS) [51]. The Al thin film was deposited on glass substrate by thermal evaporation followed by anodization to get pore diameter of $40 \mathrm{~nm}$. Further, the polyelectrolyte solutions (poly (acrylic acid) and poly (allylamine hydrochloride)) were coated on NAA substrate using layer by layer coating process to form a protective layer during buffer solution immersion process. 


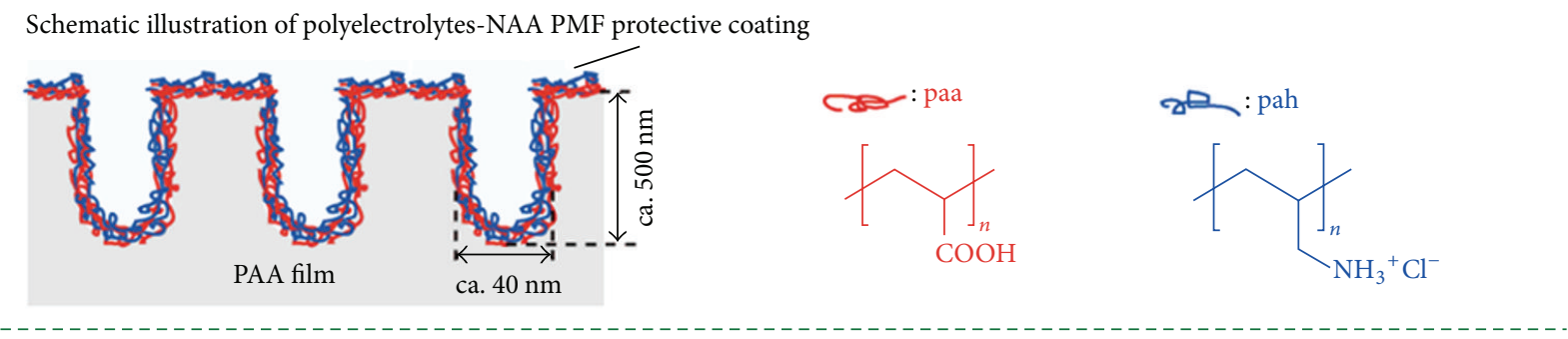

Schematic representation of DNA-DNA
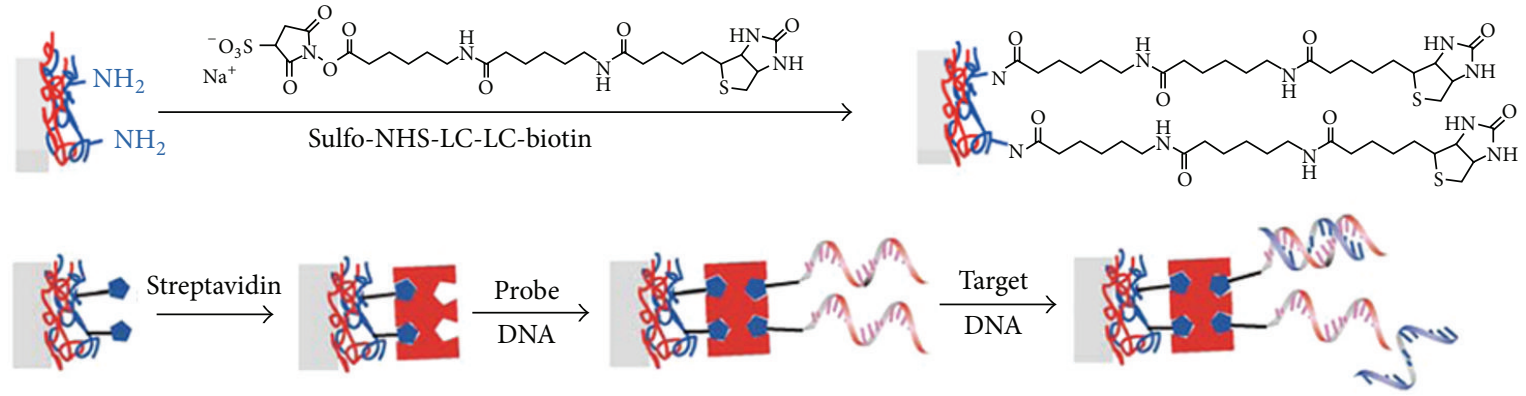

FIGURE 6: Layer by layer coating of polyelectrolytes on NAA surface and hybridization process of DNA-DNA on NAA [51].

Detection of DNA-DNA hybridization on NAA substrate by optical wave guide spectroscopy was carried out as follows: initially streptavidin was immobilized with biotin on NAA surface to detect DNA which shows increased red shift upon binding. Further, this immobilized DNA on NAA surface was bonded with noncomplementary (T2) target DNA. Thus, red shift has been increased due to complementary target DNA (T1) added with probe due to hybridization. Better sensing detection was found from complementary target DNA (T1) probe on NAA surface due to DNA hybridization which was observed using optical wave guide spectroscopy. Schematic representation of polyelectrolytes-NAA film and DNA-DNA hybridization assay using polyelectrolytes-NAA is shown in Figure 6.

4.5. Detection of Viral Particle. Moon et al. developed NAA to capture and align procapsid (phi29 viral) particles using fluorescence microscopy [87]. Al foil was anodized to prepare the diameter and interpore distances of 75 and $105 \mathrm{~nm}$, respectively. The pore structure was modified by coating of $\mathrm{Al}_{2} \mathrm{O}_{3}$ film using atomic layer deposition (ALD) with average pore diameters of $38 \mathrm{~nm}$. The surface of NAA was cleaned using 3 (trimethoxysilyl) propyl aldehyde (TMSPA/TMS) solution. Later, substrate was incubated in phi29 viral and followed by rinsing in TMS buffer solution to remove unwanted phi29 viral particles on surfaces. Later, DNA package on NAA substrate was tethered to capture phi29 viral particles through polishing and centrifugation. By polishing, the chemically modified NAA substrate was incubated in procapsid solution so that high numbers of phi29 viruses were bound into nanopores and small amounts of viruses were out of the nanopores. By centrifugation, the pore sizes were shrunk to $38 \mathrm{~nm}$ by ALD method: actual size of phi29 viral particles was $45 \mathrm{~nm}$. The attractive capture behavior was observed via centrifugation for phi29 viral particles on DNA packaged
NAA substrate. The nanopore sizes were less than viral particle sizes; thus, it was easy to be captured onto substrate. The fluorescence microscopy was used to detect the viral particles on NAA substrates.

4.6. Detection of Vitamin C. Chen et al. developed the photonic structure of biomimetic based NAA with distributed Bragg reflector for the sensing of vitamin C molecules [52]. Different anodization process was used to fabricate NAA with distributed Bragg reflectors by galvanostatic pulse anodization process. However, excellent sensing performance of vitamin $\mathrm{C}$ was observed for NAA prepared with anodization period of $675 \mathrm{~s}$ at $3^{\circ} \mathrm{C}$ with current ratio of $6: 1$ compared to other anodization parameters. Schematic representation of vitamin C molecule captured by APTES and GTA solution on NAA distributed Bragg reflectors and sensing results of vitamin $\mathrm{C}$ molecule with respect to change in effective optical thickness (OT) are presented in Figure 7. Surface functionalization of sensor involves the APTES modified NAA distributed Bragg reflectors with internal surface hydroxylated by using $\mathrm{H}_{2} \mathrm{O}_{2}$ solution, further modified with APTES using Chemical Vapor Deposition (CVD) process, and later treated with GTA solution to activate the amine groups of APTES. Thus, modified NAA distributed Bragg reflectors were used to adopt vitamin $\mathrm{C}$ molecules on internal surfaces. The RIS has been used to detect different solutions of vitamin $\mathrm{C}$ molecules on sensor surfaces with respect to change in effective optical thickness of sensor substrate.

4.7. Detection of Enzyme. NAA has been developed for the detection of glucose oxidase (GOx) using UV-vis spectroscopy by Darder et al. [88]. The pore diameter and thickness of NAA pores were in the range of $30-80 \mathrm{~nm}$ and $33-150 \mu \mathrm{m}$, respectively. The GOx was immobilized on NAA surface and was fixed between two disks; the acetate 


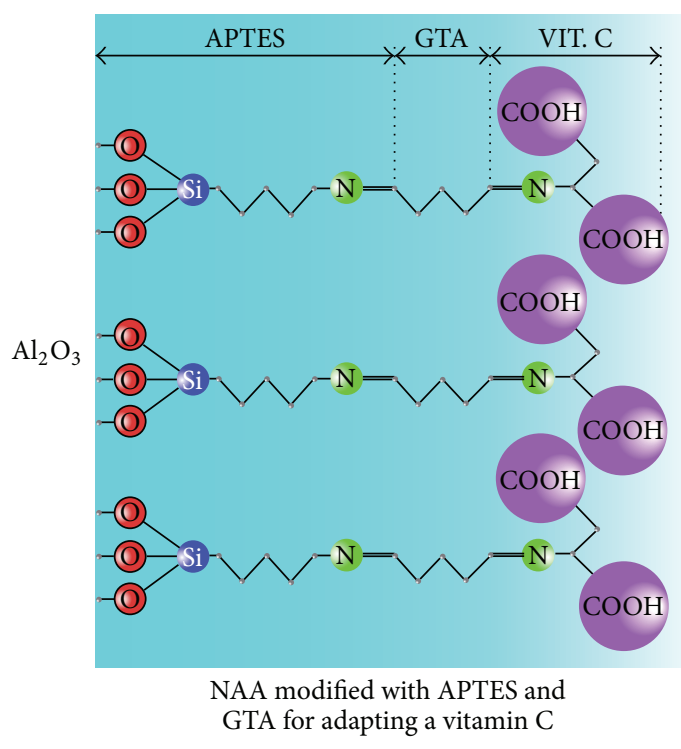

(a)

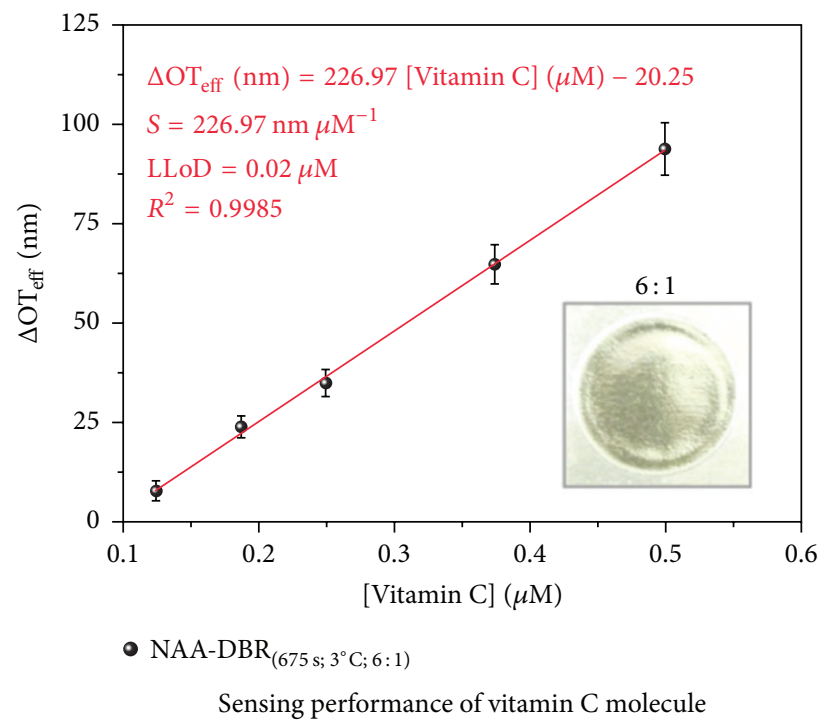

(b)

FIgURE 7: (a) NAA modified with APTES and GTA aqueous solutions for binding vitamin C molecules and (b) sensing of different concentration of vitamin $\mathrm{C}$ molecules using reflectrometric interference spectroscopy [52].

buffer solution was filtered through NAA substrate which was placed in between the disks, followed by the GOx with acetate buffer and chitosan solutions. The lack of chitosan covering on GOx modified NAA surface may decrease the enzyme activity due to leakage of glucose oxidase from the surface. Outer protecting layer of chitosan can permeate the water molecules and allows ions to transport and bind to protein. The UV-vis spectroscopy has been used to analyze the activity of immobilized GOx. The solutions of $5 \mathrm{mM}$ potassium ferrocyanide and $40 \mathrm{mM}$ glucose were mixed in $0.1 \mathrm{M}$ Tris/HCl buffer for analysis. Later, $40 \mu \mathrm{M}$ GOx was added to the prior solution for enzymatic reaction. The procedure was repeated to attain the linear relationship between the absorbance and GOx reactions. The platinum electrode holds the GOx modified NAA surface for glucose determination. The increase in pore size leads to increase in the enzymatic activity and shows good response even at low concentration. The response was very less for higher thickness NAA material with decrease in pore sizes.

Recently, polyelectrolytes based layer by layer coating on NAA for the biosensor applications was explored. Krismastuti et al. fabricated layer by layer coating of polyelectrolytes on NAA for the bacterial enzyme detection of proteinase$\mathrm{K}$ using interferometric reflectance spectroscopy (IRS) [53]. Three different thicknesses of NAA such as 1.3, 3.0, and $4.4 \mu \mathrm{m}$ with pore diameter, interpore distance, and wall thickness of $30 \pm 1.7,100 \pm 1.5$, and $70 \pm 1.8 \mathrm{~nm}$, respectively, were fabricated. The aim was to increase the thickness of porous layer to influence the easy detection of the enzyme. Polyelectrolytes like poly (sodium-4-styrenesulfonate) (PSS) and poly-L-lysine (PLL) have been used as negatively and positively charged layers, respectively, for layer by layer coating onto the sensor surface. Instead of gold coating on alumina surface polyelectrolytes were coated due to several advantages such as ease of availability and low cost and can play a vital role in increasing the RI on sensor surface. The fabrication process of layer by layer deposition of polyelectrolytes on to the sensor surface is as follows: dust free NAA was silanized by APTES, followed by 7$10 \mathrm{~nm}$ thick gold film onto NAA substrate to prevent the pore blocking. The second step is layer by layer coating of polyelectrolytes using custom designed flow cell and coating was monitored by IRS. Separately PSS and PLL were taken in $\mathrm{NaCl}$ solution: PSS solution, followed by PLL solution at a flow rate of $0.2 \mathrm{~mL} / \mathrm{min}$, was injected alternatively into the sensor surface. Thus, multilayer coating of opposite charges of polyelectrolytes was achieved on the NAA surface. The polyelectrolytes coated NAA sensors of different thickness were used to detect bacterial enzyme of proteinase- $K$ by IRS spectroscopy system which is shown in Figure 8. The enzyme exposure to substrate of thickness $1.3 \mu \mathrm{m}$ shows the effective optical thickness (EOT) of $1.9 \mathrm{~nm}$ and after washing sample with Mili-Q water the EOT was blue shifted to $7.1 \mathrm{~nm}$. Proteinase-K enzyme was also exposed to substrate of $3.0 \mu \mathrm{m}$ thick which reveals that EOT was blue shifted to $1.8 \mathrm{~nm}$ and after washing with water it was shifted to $1.9 \mathrm{~nm}$. However, proteinase- $\mathrm{K}$ exposure on the substrate of $4.4 \mu \mathrm{m}$ thick showed red shift in EOT which is due to polyelectrolytes being diffused deep inside the nanopores to form high thick sensor so that enzymes were occupied easily into porous layers and hence increasing the RI of sensor substrate, compared to those of 1.3 and $3.0 \mu \mathrm{m}$ thick sensor.

A simple and smart detection by using trypsin immobilized NAA through PL system was developed by Santos et al. [54]. NAA was fabricated by two-step anodization method; the average pore size and lengths were of $40 \mathrm{~nm}$ and $7.1 \mu \mathrm{m}$, respectively. The size of trypsin biomolecule was $3.8 \mathrm{~nm}$ and hence molecules were easily accommodated and immobilized 


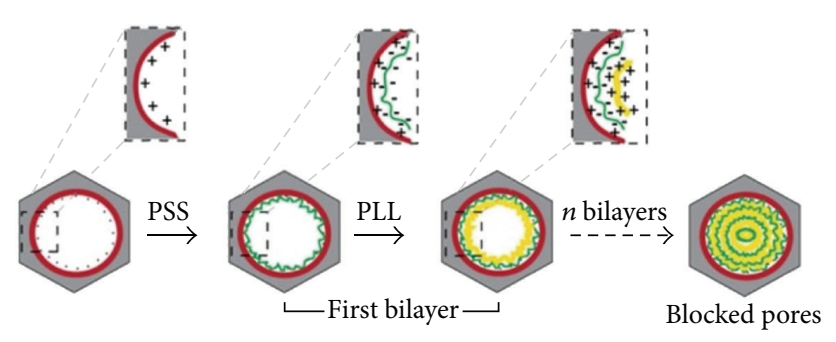

(a)

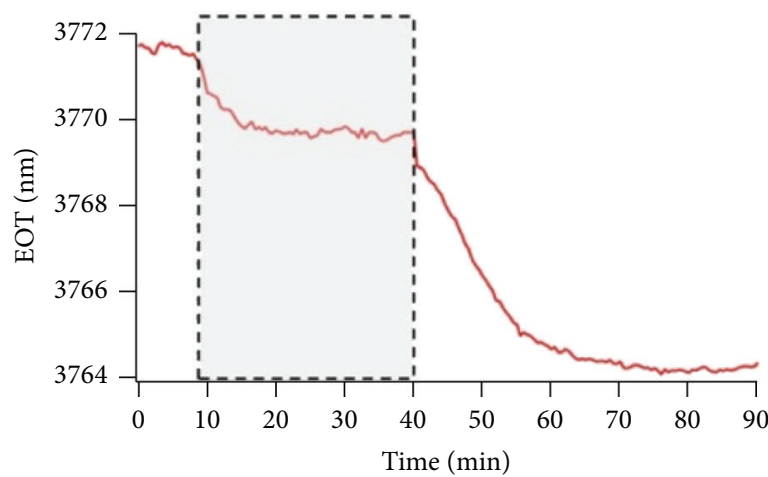

(i)

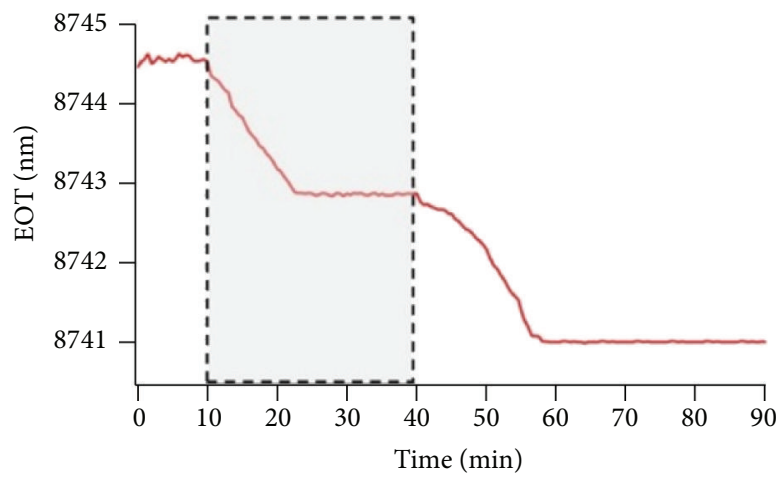

(ii)

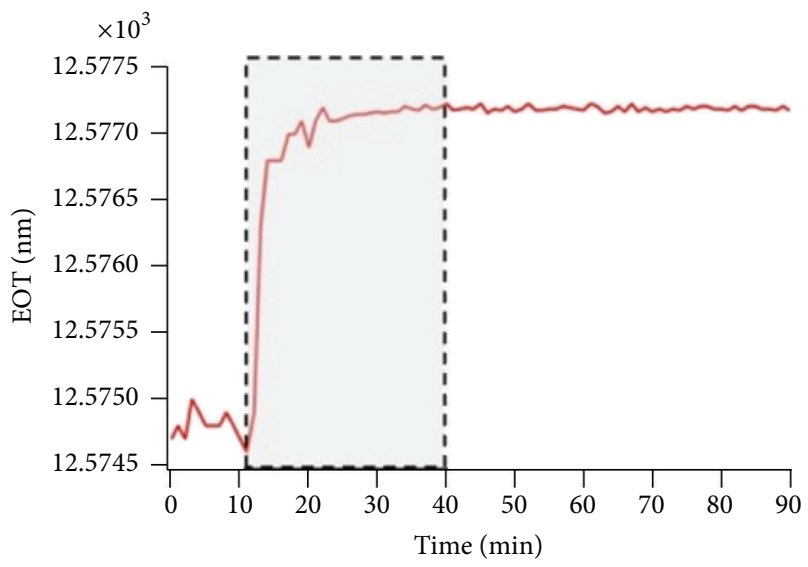

(iii)

(b)

FIGURE 8: (a) Layer by layer deposition using polyelectrolytes like poly(sodium-4-styrenesulfonate) (PSS) (greenish) and poly-L-lysine (PLL) (yellowish) on NAA and (b) the effective optical thickness (EOT) plot of sensing of proteinase-K by layer by layer coated polyelectrolytes on NAA thicknesses of 1.3 (i), 3 (ii), and (iii) $4.4 \mu \mathrm{m}$ using IRS system [53].

on the nanopores. The process of trypsin immobilization in nanopores was made possible by silanization process: APTES and glutaraldehyde (GTA) were mixed together with NAA, and later trypsin biomolecules were allowed to bind via amino groups on porous substrate by the incubation process. The schematic diagram of different stages of immobilized trypsin molecules on NAA and sensitivity plot of trypsin by change in effective optical thickness with respect to different stages on NAA surface by PL spectroscopy is shown in Figure 9. The detection of immobilized trypsin has been done by observing change in effective optical thickness on sensor substrate by PL. Effective optical thicknesses of bare NAA, APTES coated NAA, GTA-modified NAA, and immobilized trypsin on NAA were found to be $0.1,0.6$, 1.2 , and $1.8 \%$, respectively. From the above observation, it has been confirmed that there was an obvious increment in effective optical thickness of NAA.

Invertase is one type of enzyme which is used for the production of sugars and used for hydrolysis process like inverting sucrose into glucose and fructose. Thus, NAA biosensor was fabricated and applied to real-time detection of invertase enzyme using Surface Plasmon Resonance (SPR) sensor by Dhathathreyan [55]. For the enzyme immobilization, the substrate of pore diameter and interpore distances of 60 and $100 \mathrm{~nm}$, respectively, were used. The uncontaminated NAA substrate was kept with invertase enzyme solution 


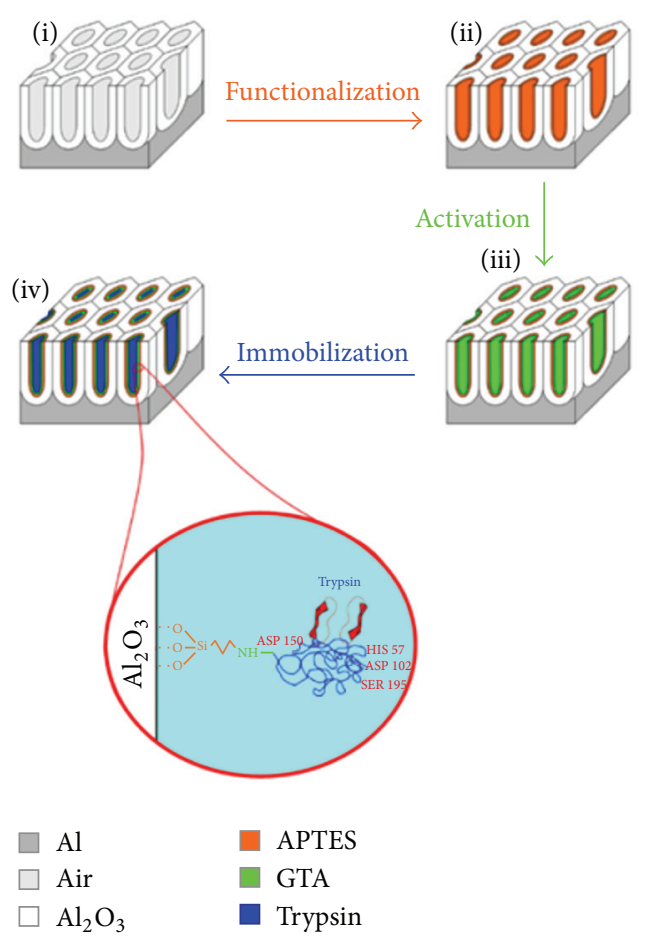

(a)

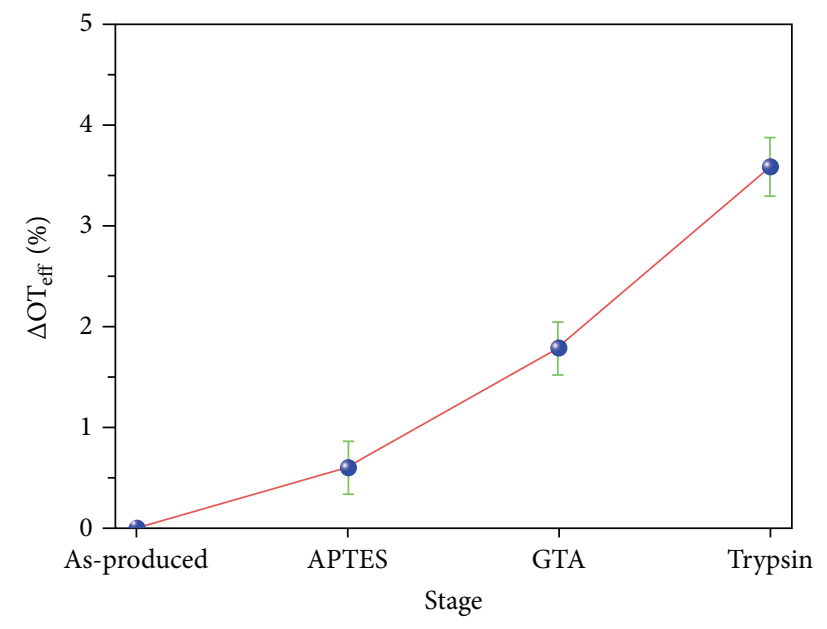

(b)

FIGURE 9: (a) The schematic representation of immobilization of trypsin on NAA, the different stages of the fabrication process of the NAA enzymatic sensor. (i) Bare NAA, (ii) APTES modified NAA, (iii) GTA-modified NAA, and (iv) trypsin immobilized NAA. (b) The effective optical thickness with respect to different surface functionalization [54].

for $1 \mathrm{~h}$ at required $\mathrm{pH}$ value. The enzyme immobilization activity was tested in the experimental flow cell by gold coated glass plate and SF10 prism set-up sealed with Teflon cell to impound tested solution. The immobilized invertase and its interaction with sucrose were analyzed by calculating the change in RI on substrate using SPR signal as shown in Figure 10.

On the other hand, gelatin-modified NAA was fabricated for the real-time detection of trypsin using RIfS by Nemati et al. [56]. Gelatin molecules play vital role for chemical sensing and better selectivity towards trypsin molecules. The NAA substrate was gold coated with $5 \mathrm{~nm}$ thickness using sputter deposition, and the APTES and GTA solutions were used to modify the surface of NAA for the formation of amino groups. After this, gelatin molecules were allowed to immobilize on inner surface of the pores. The different concentrations of trypsin enzyme $\left(0.125,0.3,0.5,0.6\right.$, and $\left.1 \mathrm{mg} \mathrm{mL}^{-1}\right)$ were used to analyze the sensing performance by using RIfS system. The effective optical thickness was changed with respect to different concentration of trypsin molecules. However, higher concentration of trypsin molecules $\left(1 \mathrm{mg} \mathrm{mL}^{-1}\right)$ showed fast response in RIfS. More concentration of gelatin molecules immobilized on the pores provides faster response to trypsin molecules. Hemoglobin has been used instead of gelatin as a chemical sensing element that also shows faster sensing performance. Schematic diagram of enzyme detection by gelatin-modified NAA using RIfS set-up and also different concentration of analyte solution leads to

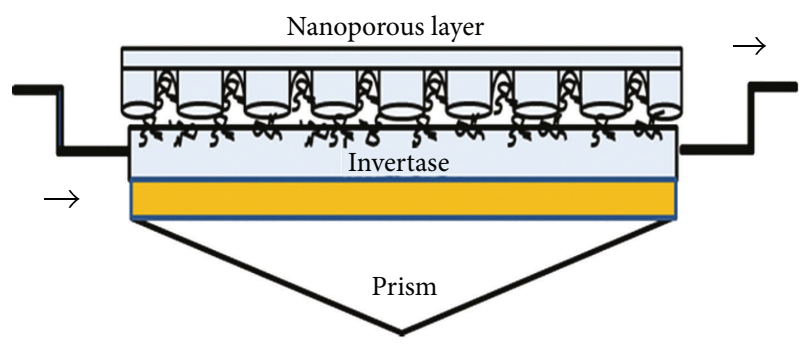

FIGURE 10: Sensing of immobilized invertase enzyme on NAA surface in flow cell of SPR [55].

change in effective optical thickness in real-time which is shown in Figure 11.

\section{Gold Coated NAA (Au-NAA) Biosensor}

5.1. Detection of Protein. While integrating NAA into the SPR system, the RI at the close proximity of the sensing platform plays vital role. Thus, Hierro-Rodriguez et al. have extracted the RI values of different NAA templates which are useful in developing SPR and long range SPR sensors [89]. Similarly, Kim et al. developed an Au-NAA biosensor for the label-free detection of aptamer-protein binding interaction on sensor surface using combination of localized surface plasmon resonance (LSPR) and interferometry [90]. The obtained uniform porous structured NAA substrate had pore diameters and 


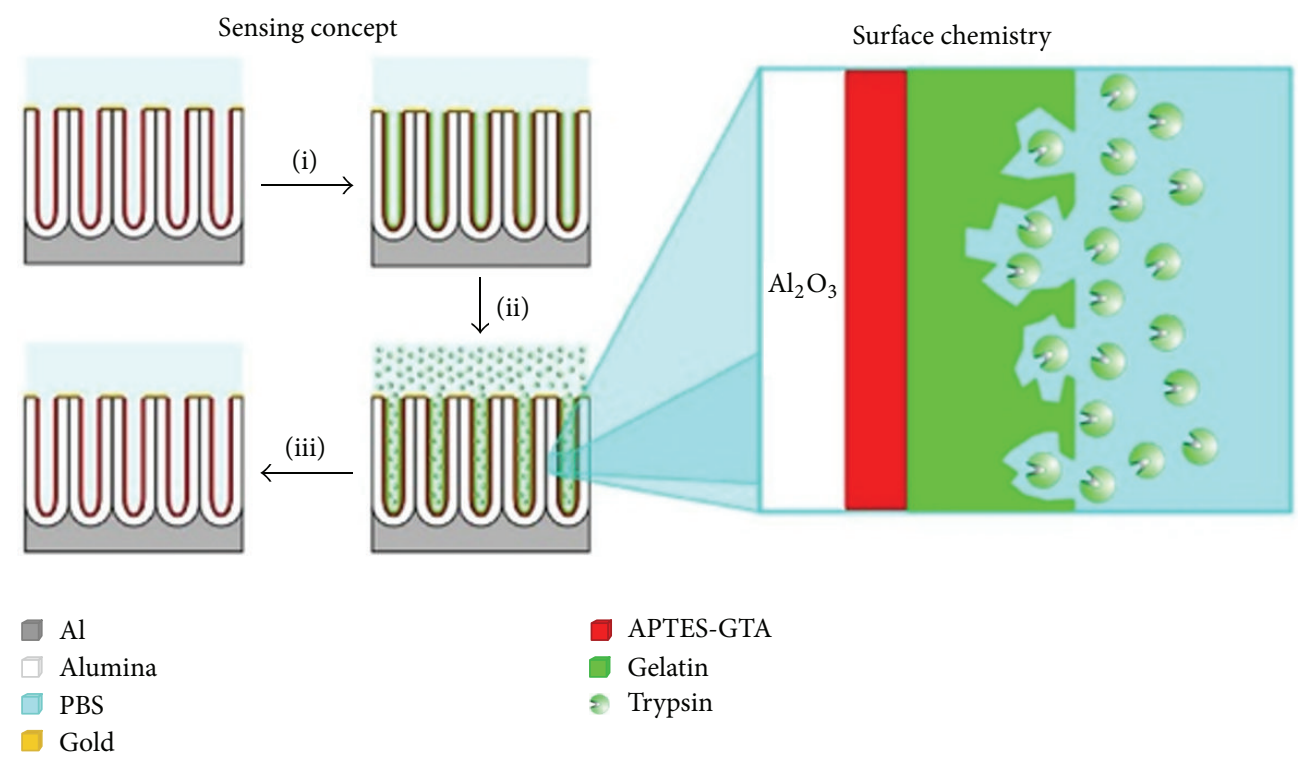

(a)

(b)

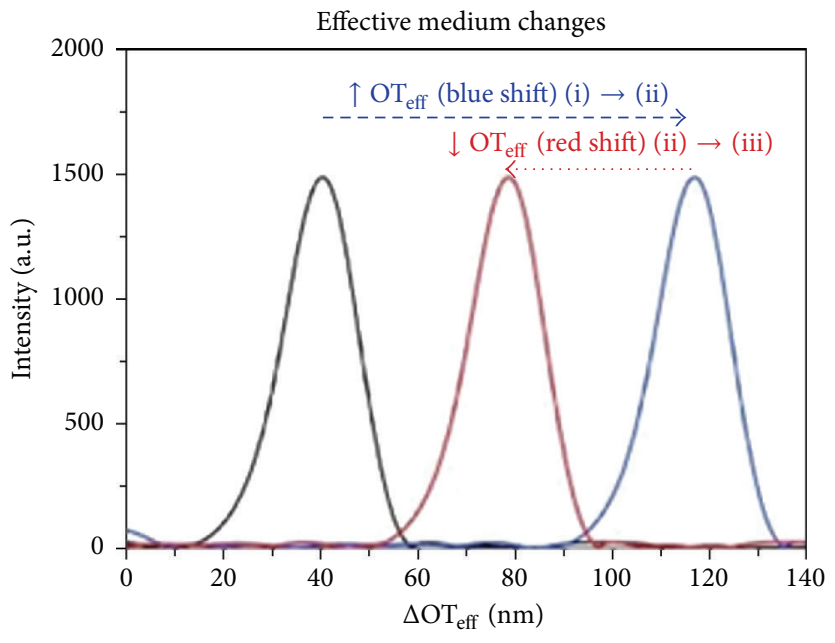

$\begin{array}{ll}\text { APTES-GTA NAA-PF } & \text { Gelatin-modified NAA-PF }\end{array}$

(c)

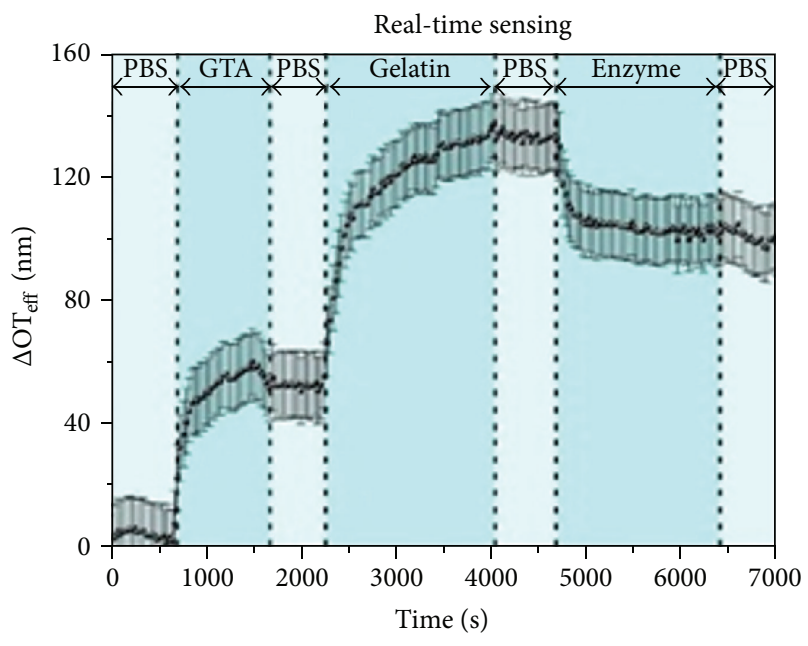

(d)

FIGURE 11: (a) Sensing of enzymes by gelatin-modified NAA using RIfS system, (b) surface chemistry used in enzymatic sensor where gelatin acts as the chemical sensing molecule for the enzyme, (c) the effective optical thickness changes in gelatin modified NAA with respect to the intensity using RIfS, and (d) real-time sensing of enzyme [56].

interpore distances of 60 and $120 \mathrm{~nm}$, respectively. The size of pore diameter was reduced to $47 \mathrm{~nm}$ by coating of $\mathrm{Au}$ thin film on NAA surface by thermal evaporation technique with thickness of $15 \mathrm{~nm}$ for the enhancement in optical properties on their surface. The aptamer-I was immobilized on NAA surface by rinsing NAA with thiolated aptamer-I and 6-mercaptohexanol solutions. Further, aptamer-I modified NAA substrate was rinsed with mixture of thrombin and PBS solution. After processing, aptamer-II solution was interacted with aptamer-I-thrombin modified NAA surface. The RI was high for aptamer-I with NAA compared to bare $\mathrm{Au}$ NAA. The obtained high concentration of aptamer-I was immobilized on Au-NAA which was red shifted and was observed through interferometry system. Later, aptamer-Ithrombin immobilized Au-NAA surface revealed that the red shift was due to enhancement in RI of NAA. However, nonspecific binding of aptamer-I and aptamer-II on $\mathrm{Au}$ NAA surface was observed whereas aptamer-I-thrombin on Au-NAA surface resulted in better binding interaction. The RI depends on the binding interaction of biomolecules on sensor surfaces. The saturation state was attained for the concentration of thrombin more than $100 \mu \mathrm{M}$; above this concentration no response was found from interferometry and LSPR signals. 


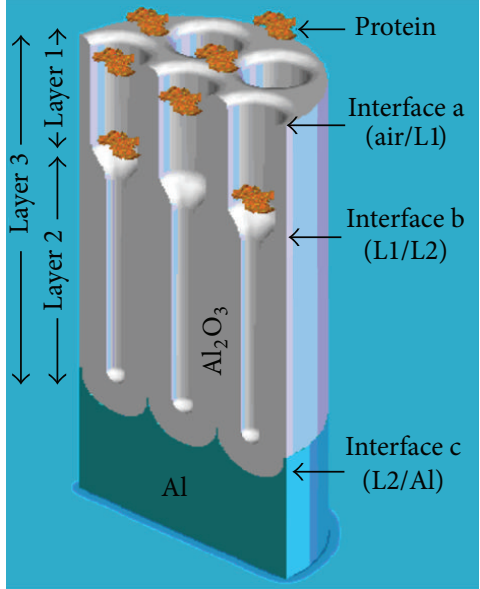

(a)

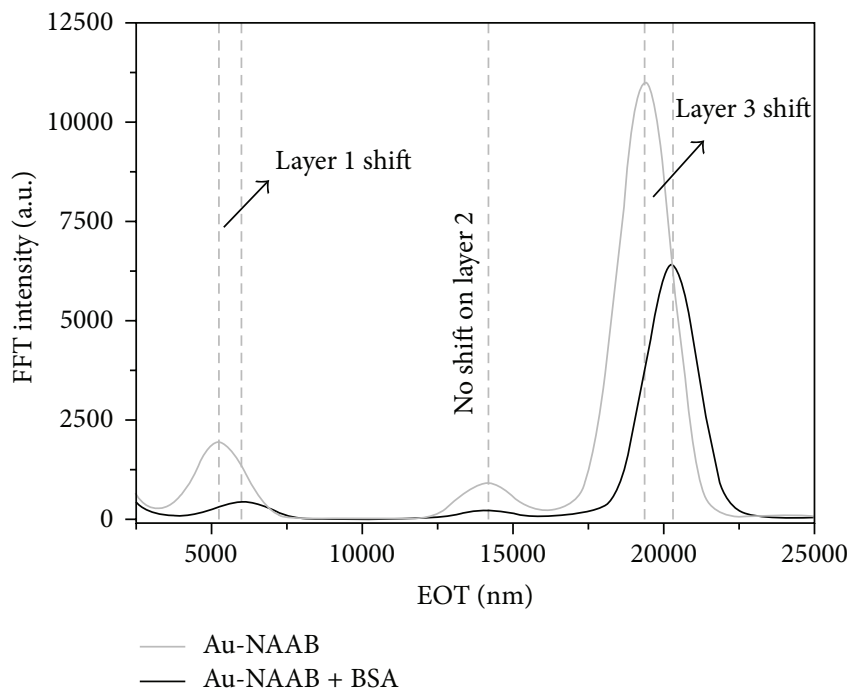

(b)

FIgURE 12: (a) The schematic diagram of Au-NAA bilayer structure and (b) effective optical thickness with and without protein on Au-NAA bilayer optical biosensor measured by fast Fourier transform [16].

In recent years, there have been increased interest on developing label-free optical biosensors based NAA. These types of label-free optical biosensors are exhibiting high throughput in medicinal applications. The Au-NAA bilayer substrate as label-free optical biosensor for BSA has been developed by Macias et al. [16]. The bilayer structure of NAA has been prepared with different porosity with pore diameter and depths of $68 \mathrm{~nm}$ and $1.9 \mu \mathrm{m}$ as well as bottom layers with $33 \mathrm{~nm}$ and $4.7 \mu \mathrm{m}$, respectively. The design of bilayered structures was planned in such a way that the top layer can be used as entry for protein whereas low pore size bottom layer could not allow protein in this direction. The gold deposition on NAA was achieved by coating $10 \mathrm{~nm}$ thickness of gold film on top of the surface of NAA bilayer via sputtering technique. The greater sensitivity was attained by increasing the layer of gold film on sensor surface which increases the RI of NAA. Initially, sensor materials were incubated with proteins at quantity of $1 \mathrm{mg} / \mathrm{mL}$ in PBS buffer solution for $2 \mathrm{~h}$ at $5^{\circ} \mathrm{C}$ to avoid the binding on surface of gold layer. Further, samples were thoroughly washed in PBS solution to remove any free contaminants. Thus, identical procedure was carried out for protein in PBS solution for biosensing applications. It was found that the protein adsorption occurred on the surface of NAA due to electrostatic forces between protein and sensor substrate. The allowed protein was detected at the top layer using change in optical thickness between two layers. The following equation has been used to detect the protein by change in optical thickness: that is,

$$
\begin{aligned}
\Delta \mathrm{EOT}= & \text { EOT }(\text { after protein penetration }) \\
& - \text { EOT (before protein penetration), }
\end{aligned}
$$

where EOT is effective optical thickness.

The schematic diagram of fabrication and structure of $\mathrm{Au}-$ NAA bilayer and fast Fourier transform graph of the optical biosensing response of Au-NAA bilayers before and after the penetration of protein into sensor structures are shown in Figure 12. As shown in Figure 12(b), the peak intensity was very less in overall gold coated NAA bilayer with infiltration of protein due to adsorption of protein in layer 1 . The increase in effective RI of layer 1 will decrease the RI of overall sensor substrate. The effect of optical thickness was increased in layers 1 and 3 due to the above reason, whereas less shift in layer 2 optical thickness was observed. The same results were obtained for Au-NAA bilayer without protein where higher intensified peaks were formed. The Au-NAA bilayer with the protein (BSA) has been sensed by intensified peaks of layers 1 and 3.

In similar work, Yeom et al. fabricated Au-NAA for real-time detection of antigen (C-reactive protein) [57]. The fabricated NAA have dimensions of $100 \mathrm{~nm}$ pore diameter and depths of pores were $4.9 \mu \mathrm{m}$. The Au-NAA materials were obtained by electron beam evaporation method. Here, gold layer coating on sensor material acts as high SPR compared to bare $\mathrm{Ag}$ or $\mathrm{Au}$ metals. The Au coated sensor substrate has produced LSPR and it plays vital role in accepting and immobilizing antibody on sensor surfaces. The immobilized antibody has been captured by Au-NAA material which was rinsed and kept for $24 \mathrm{~h}$ in solution of 11-mercapto-1undecanol and $\mathrm{EtOH}$. The unwanted antibody was removed by treating sensor surface with Tris- $\mathrm{HCl}$ solution instead of PBS solution to avoid the damage. The detection of antigen using Au-NAA works on LSPR principle in which the allowed antigens react with antibody to change RI of the sensor substrate. The biosensing mechanism has been predicted by change in the reflectivity of the sensor substrate by real-time biosensor detection method (Figure 13).

Similarly, Hiep et al. fabricated photonic structure of Au-NAA film for the detection of biomolecular interaction using interference localized surface plasmon resonance 


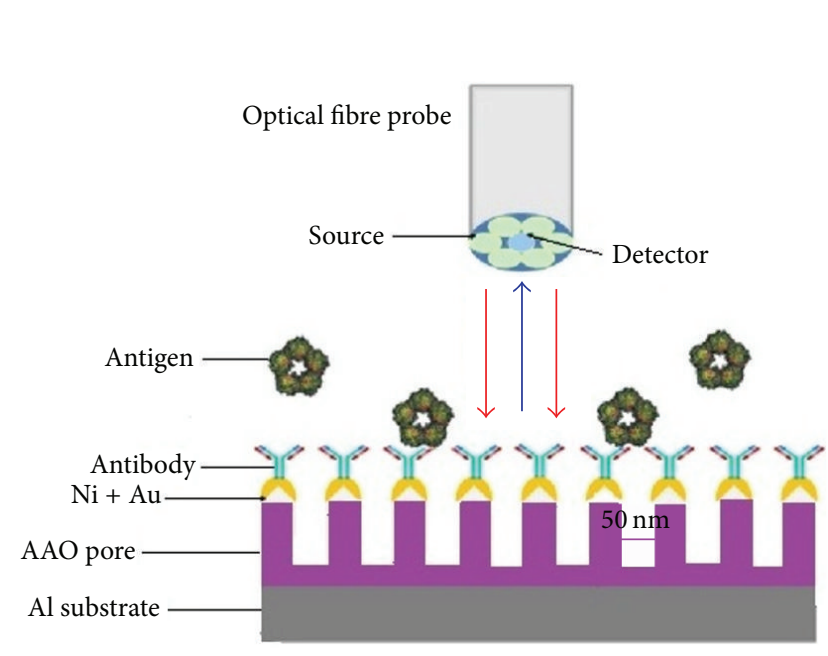

(a)

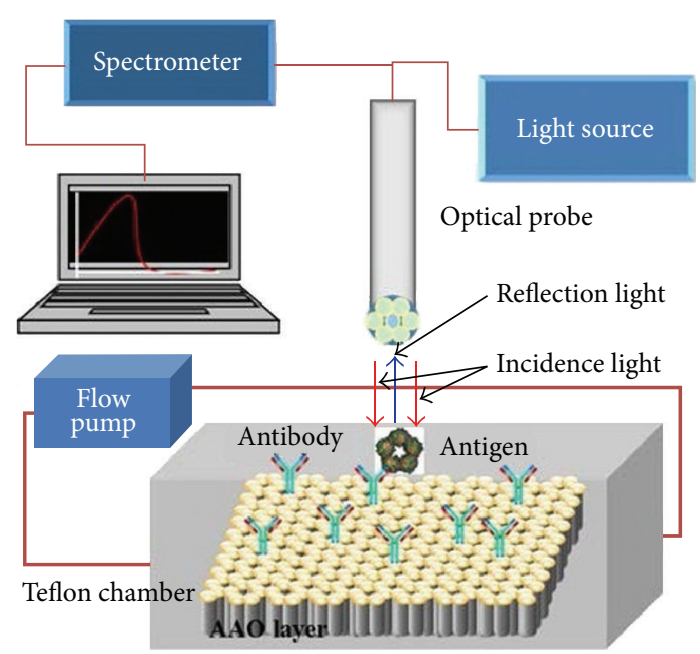

(b)

FIgURE 13: (a) The antigen detection using antibody functionalized Au-NAA and (b) set-up for the real-time detection of antigen [57].

(iLSPR) system [58]. The gold coated NAA film (Au-NAA) has thickness of $270 \mathrm{~nm}$ which was rinsed in 11-amino-1undecanethiol hydrochloride solution to functionalize with free amino groups available at substrate surface. A mixture of PBS and BSA solutions was used for rinsing NAA surface. The iLSPR system was used for the detection of binding interaction of two different biomolecules on NAA surface. The RI was slightly changed due to interaction of biotinavidin binding on NAA surface and further increased for BSA binding with avidin on sensor surface. The limit of detection for avidin using Au-NAA film by iLSPR was found to be $100 \mathrm{ng} / \mathrm{mL}$. Schematic diagram shows iLSPR biosensor and wavelength depends on reflectance spectra changes by biotin-avidin and biotin-BSA binding interaction on $\mathrm{Au}-$ NAA surface (Figure 14).

5.2. Detection of DNA. Kim et al. fabricated "cap like" gold structures which were coated on NAA for the detection of DNA hybridization sensing mechanism using LSPR microscopic system [59]. The fabricated NAA has the diameter and thickness of $60 \mathrm{~nm}$ and $5 \mu \mathrm{m}$, respectively. The immobilization of DNA on Au-NAA was achieved by mixing of $10 \mu \mathrm{M}$ thiolated DNA probe and $100 \mu \mathrm{M}$ 6-mercaptohexanol solutions which were further applied on sensor surface. A target DNA was hybridized with probe DNA on sensor surface by PBS solution. The sensing of probe DNA and target DNAs hybridization was captured by the increment in wavelength shifts $\left(\lambda_{\max }\right)$ and relative reflected intensity which depends on change in the concentration of target DNA on the sensor surface. The saturation state was found at the concentration of target DNA which exceeded the limit of $10 \mu \mathrm{M}$. Schematic diagram shown in Figure 15 represents the working mechanism and sensing measurement of DNA hybridization on Au capped structure of NAA using LSPR system.

5.3. Detection of Cells. In biosensors field, researchers are trying to develop NAA with gold film coating for the detection of biomolecules such as proteins and DNA. However, less study has been carried out on the fabrication of Au-NAA for the detection of single living cell adhesion and seeding on sensor surfaces using SERS system. Toccafondi et al. fabricated biosensor substrate, that is, Au-NAA, for single cell sensing with the help of SERS [91]. Fabricated Al thin film on glass substrate has thickness of $500 \mathrm{~nm}$ by e-beam evaporator followed by anodization process. The prepared film structure had pore diameter and depth sizes of 50 and $120 \mathrm{~nm}$, respectively. Thick gold layer of $25 \mathrm{~nm}$ on NAA was coated by e-beam evaporator and $\mathrm{N} 2$ a cell lines were used from mouse neuroblastoma for living cell sensing application by bare NAA and Au-NAA using SERS system. In this experiment, $5 \times 10^{4} \mathrm{~N} 2$ a cells were mixed in $2 \mathrm{~mL}$ of high glucose medium and cells were grown in this solution at $37^{\circ} \mathrm{C}$ with humidified $\mathrm{CO}_{2}$ atmosphere. Later, cells were allowed for sensing application using bare NAA and Au-NAA. During the sensing process, different diameters (60 and $120 \mathrm{~nm})$ of bare NAA were used to adhere to the N2a cells without using any adhesion solution which was due to biocompatibility and surface roughness of the material. The adhered cells were captured with fluorescent dyes by confocal fluorescence microscopy. However, the aim was to develop Au-NAA for living cell sensing applications using SERS. The excellent micro Raman scattering improvement has been achieved by cell seeded on Au-NAA. The sensing of Au-NAA material was done by laser light obtained from micro Raman spectroscopy. Enhancement in Raman peaks was observed in the presence of N2a cell due to adhesion and growth on surface of sensor material. On the other hand, Toccafondi et al. fabricated biocompatible Au-NAA for the detection of fibroblast living cells using SERS and fluorescence microscopy [92]. The SERS signals were high for the higher pore dimension. The cells were grown on Au-NAA surface due to biocompatibility of material. The living cells (NIH-3T3 cells) were incubated in Au-NAA surfaces for 2 days and grown on the surface. The better sensitivity was achieved for the bigger pores $(130 \mathrm{~nm})$ 

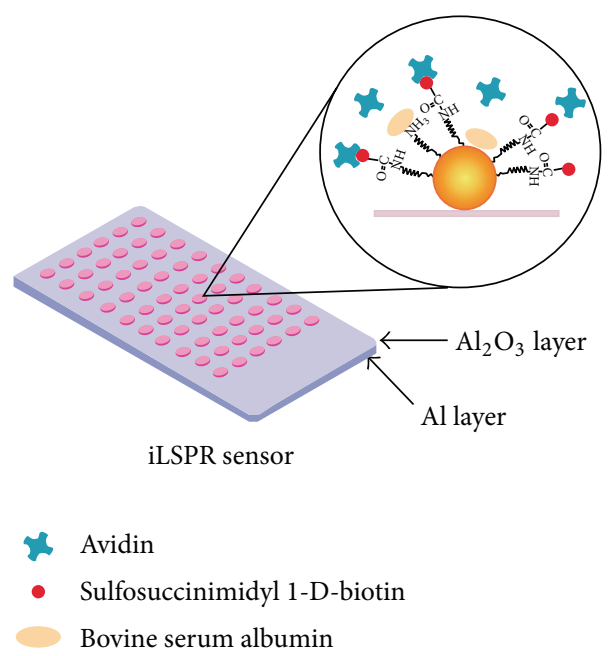

(a)

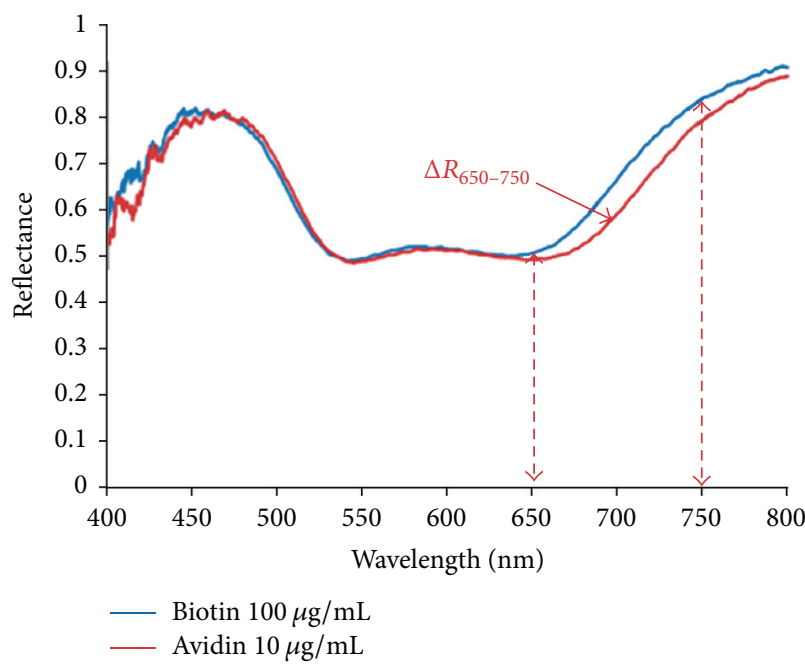

(b)

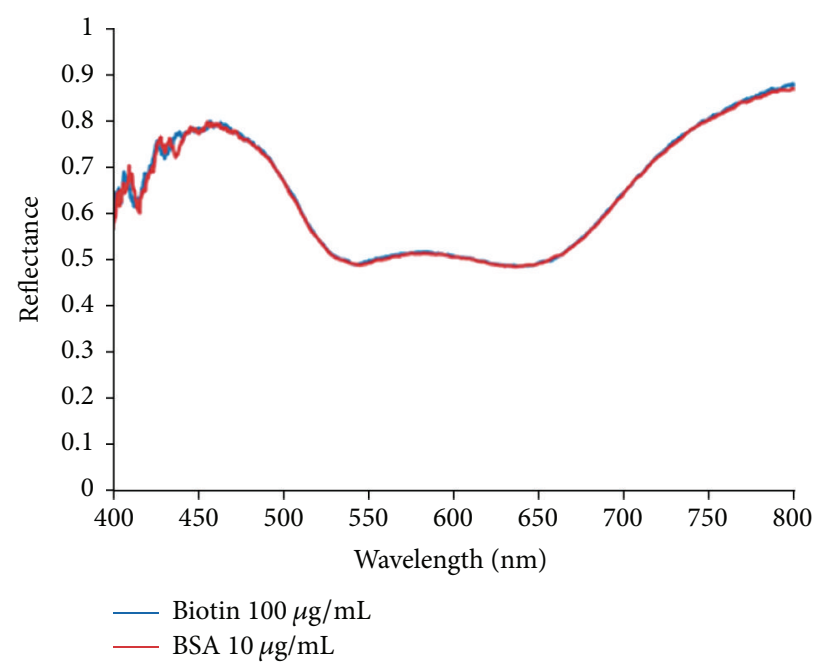

(c)

FIGURE 14: (a) Schematic representation of the iLSPR biosensor, (b) the reflection spectra which depend on the change in RI upon binding of biotin-avidin, and (c) interaction of biotin-BSA [58].

and smaller pore lengths $(50 \mathrm{~nm})$ of Au-NAA for living cell sensing using SERS system.

5.4. Detection of Fluorescent Molecules. Das et al. developed novel Au-NAA with honeycomb pore structure for the biosensor applications with fluorescent molecules such as cresyl violet, rhodamine 6G, and green fluorescent proteinmut2 using SERS system [60]. Honeycomb pore structures of NAA with the pore size and wall thickness of 15 and $36 \mathrm{~nm}$, respectively, were fabricated. Three different honeycomb pore structures were fabricated: NAA1 with pore size and wall thicknesses of $181 \pm 45$ and $82 \pm 35 \mathrm{~nm}$ (bigger pores), NAA2 of $82 \pm 7$ and $21 \pm 7 \mathrm{~nm}$ (medium pores), and NAA3 of $29 \pm 4$ and $13 \pm 4 \mathrm{~nm}$ (smaller pores), respectively. The SERS signals were enhanced by coating $25 \mathrm{~nm}$ of gold film on NAA by thermal evaporation, namely, Au-NAA1 with pore size and wall thickness of $157 \pm 35,104 \pm 35 \mathrm{~nm}$, and Au-NAA2 with $59 \pm 9,40 \pm 9 \mathrm{~nm}$, and Au-NAA3 with $15 \pm 3$ and
$36 \pm 3 \mathrm{~nm}$, respectively. To achieve the sensing, fluorescent molecules were deposited on different Au-NAA substrates by chemisorption method and tested with the molecules of concentration $10^{6} \mathrm{M}$. Further, cresyl violet, an organic dye, was chosen which is routinely used in nerve tissue sector for biological stain. At first, cresyl violet molecules were coated on different Au-NAA structures revealing that SERS signals were enhanced due to $\mathrm{NH}_{2}$ groups which were forcefully attached on sensor surfaces attributed to vibrational modes at $591 \mathrm{~cm}^{-1}$. These molecules were strongly attached on surface of Au-NAA3 due to low porosity whereas in case of Au-NAA1 and Au-NAA2 molecules were not attached due to medium and high porosity, respectively. It was found that the enhancement in SERS measurement of Au-NAA2 compared to other two substrates due to low pore size and wall thickness. The enhancement was observed in SERS measurements for rhodamine $6 \mathrm{G}$ and green fluorescent protein-mut2 molecules which was due to the extensive 


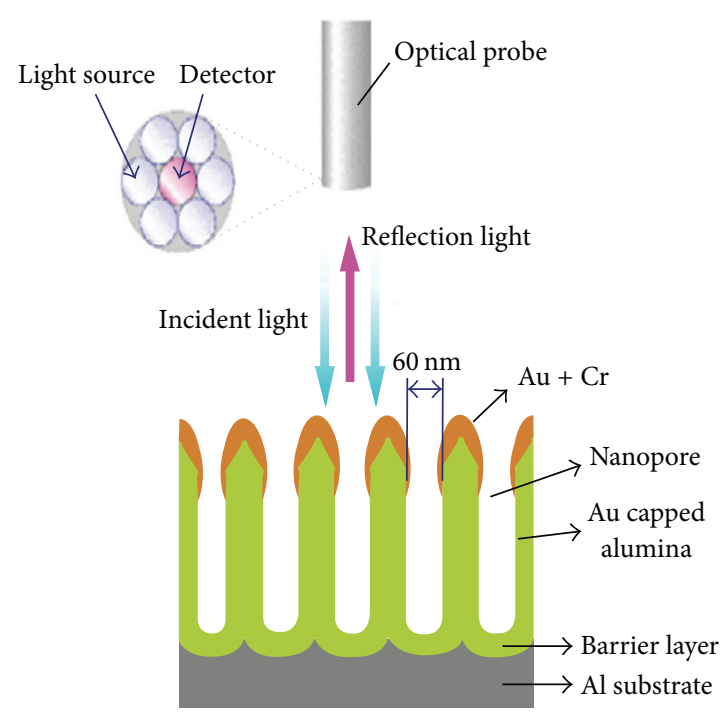

(a)

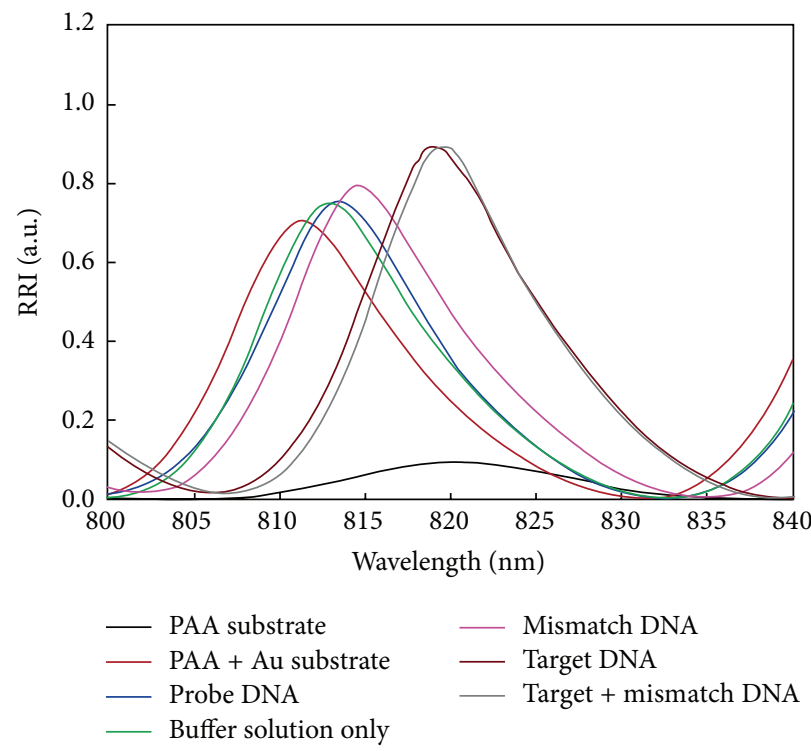

(b)

FIGURE 15: (a) DNA hybridization sensing mechanism of Au capped structure of NAA; (b) change in relative reflected intensity (RRI) of DNA hybridization detection on Au capped structure of NAA [59].

use of rhodamine $6 \mathrm{G}$ as a dye molecule in fluorescence microscopy. Due to the fact that molecular adsorption on metal surfaces is because of electronegativity of metals, this electronegativity is high for noble metal Au compared to $\mathrm{Ag}$. Thus, the adsorption of molecules was enhanced by $\mathrm{Au}$ coating on sensor substrates. The rhodamine $6 \mathrm{G}$ molecules were deposited over surfaces of Au-NAA1 and Au-NAA2 to test in SERS systems. These molecules were strongly bound to Au surfaces and the enhancement in SERS was observed with strong intensity of vibrational modes at 1361 and $1510 \mathrm{~cm}^{-1}$. The green fluorescence protein-mut2 was a fluorescent protein which has a capability of fluorescence recovery to use in slow biological processes. The SERS spectra enhanced through the fluorescent proteins which were coated on Au-NAA2 surfaces and SERS peaks were obtained at $1500 \mathrm{~cm}^{-1}$. The schematic diagrams of honeycomb nanopore structure of Au-NAA and the SERS spectra are shown in Figure 16 which reveals the detection of (i) cresyl violet and (ii) rhodamine $6 \mathrm{G}$ using SERS.

5.5. Detection of Binding of Alkyl Thiol. Previously, several articles show developing of NAA for the detection of immobilized biomolecules on sensor surface by soaking the substrate in mixer of preferred buffer solutions like PBS/Tris- $\mathrm{HCl}$ containing biomolecules. However, biomolecules sometimes may not properly bind on the substrate with this technique. On the other hand, the biomolecules can also be immobilized onto the substrate by flowing the biomolecules taken in a mixture of buffer solutions within microfluidic channels for better binding due to higher chances of biomolecules coming in contact with the surfaces. The microfluidic system comprising sensor substrate has the advantages such as healthy binding biomolecule, less amount of sample for the detection, and rapid sensitivity in microchannels. For the first time Kumeria and Losic developed Au-NAA comprising microfluidic channel for label-free optical biosensing for the detection of binding of alkyl thiol on sensor substrate by enhancement of reflectivity in interferometric spectroscopy signals [61]. Pore size and pore depths of fabricated NAA structures were of $60 \mathrm{~nm}$ and $4 \mu \mathrm{m}$, respectively. In addition to that, scanning electron microscopy was used to measure the interpore distance and it was about $60-100 \mathrm{~nm}$. The reflectance interferometric spectroscopy (RIS) signals were enhanced by the pores and surface area of internal pore structures of NAA. The fabricated controlled parameter of NAA has been coated by different metals like $\mathrm{Au}, \mathrm{Ag}, \mathrm{Cr}, \mathrm{Pt}$, and $\mathrm{Ni}$ by atomic layer deposition. However, Kumeria and Losic have not observed significant reflectance interferometric intensity signal originating from $\mathrm{Pt}, \mathrm{Ag}$, and Ni metals. The better interferometric signal has been obtained for $\mathrm{Au}$ and $\mathrm{Cr}$ metals due to enhancement of reflectivity on these thin metals. The optical biosensing performance was performed only by $\mathrm{Au}$ NAA structure because Au metal has biocompatibility and chemical stability and is preferred for particular molecules binding detection for biosensing applications. The set-up comprises Au-NAA into microfluidic device with connected syringe pump and fiber optic probe. The optical biosensing showed by $1 \mathrm{mM}$ thiol molecules which were passed into Au-NAA substrate mounted microfluidic chip reveals higher wavelength with respect to change in effective optical thickness obtained for adsorption of molecules on Au surfaces. Furthermore, even at low concentration of molecules it has shown better sensitivity. The effective optical thickness has been increased with increase in the concentration of thiol molecules on sensor surfaces. They also analyzed real-time sensing performance of the molecule bounded with thiol group on sensor substrate which shows excellent maximum wavelength. The schematics shows the Au-NAA embedded 


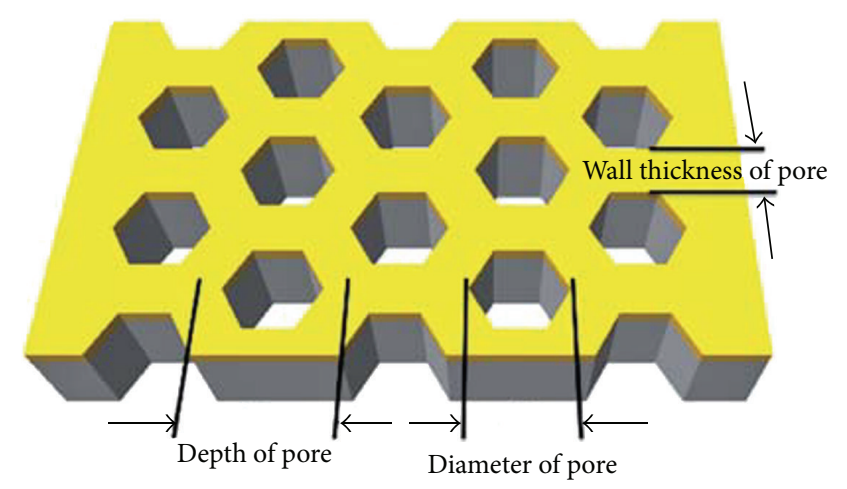

(a)

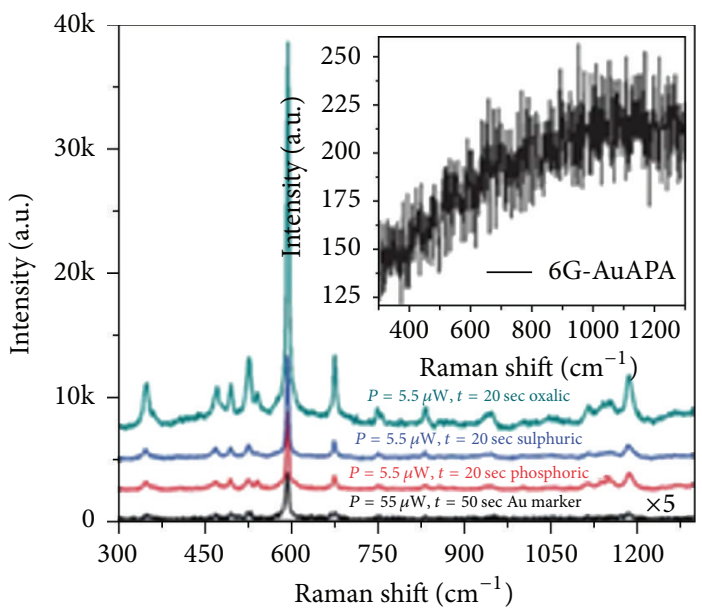

(i)

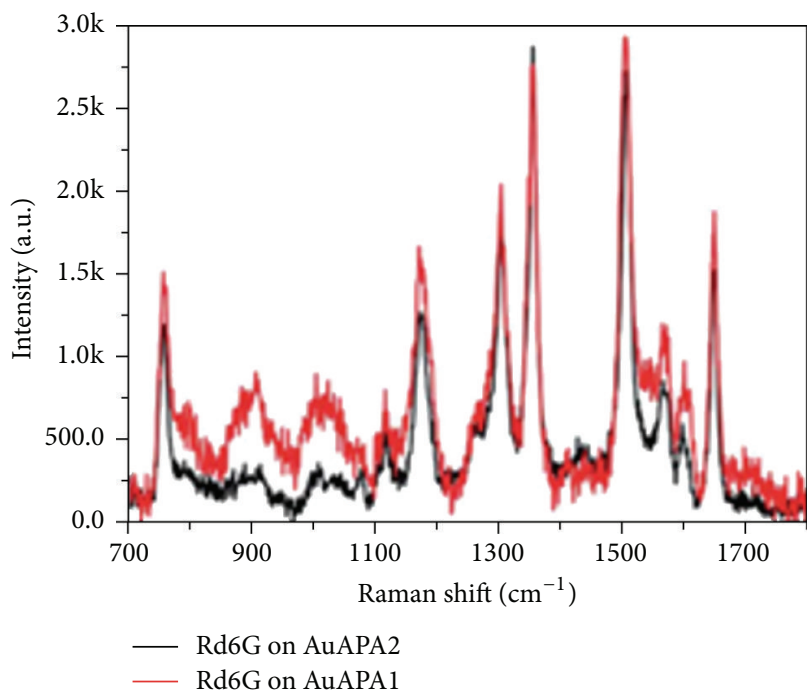

(ii)

(b)

FIGURE 16: (a) Honeycomb pore structure of Au-NAA; (b) the SERS spectra for the detection of (i) cresyl violet and (ii) rhodamine 6G [60].

in microfluidic channel for detection of alkylthiol binding by optical biosensor using RIS system (Figure 17).

5.6. Detection of Circulating Tumor Cells. Kumeria et al. developed label-free microfluidic channel based biosensor on $\mathrm{Au}-\mathrm{NAA}$ for the detection of circulating tumor cells (CTC) by reflectrometric interference spectroscopy [93-96]. Biotinylated anti-EpCAM was immobilized on the NAA surface through multiple chemical steps followed by integrating the porous NAA substrate into the microfluidic device. Spiked tumor cells in PBS and whole blood samples were injected into the device and captured with high efficiency by using interferometric spectroscopy. The fabricated NAA has pore diameter and lengths of $32 \pm 3 \mathrm{~nm}$ and $4 \pm 0.2 \mu \mathrm{m}$, respectively. The sensor surface was coated with ultra-thin Au layer of $8 \mathrm{~nm}$ by thermal vapor deposition technique. Multistep surface modification was adopted: Au-NAA were soaked in $10 \mathrm{mM}$ solution of mercaptoundecanoic acid (MUA) in order to bind it covalently onto the sensor surface, followed by soaking in solution containing streptavidin molecules at $100 \mu \mathrm{g} / \mathrm{mL}$ concentration where streptavidin binds to MUA.
Further, the streptavidin functionalized substrate was incubated in $50 \mu \mathrm{g} / \mathrm{mL}$ of anti-EpCAM antibody in PBS buffer solution. The PMMA based microfluidic chip was obtained by computer numerically controlled (CNC) micromachining method. This microfluidic chip was formed with two channels with sensing platform for Au coated NAA substrate on the glass substrate. The working principle of detection involves the use of white light emitted from optical fiber probe which was focused on anti-EpCAM modified sensor substrate incorporated in the chip and light was reflected back from the surface into the same optical fiber probe system. The change in effective optical thickness on sensor surface was attributed to the change in RI of the surface. The RIS signals were obtained after the flow of CTCs into the channels, due to binding of CTCs onto anti-EpCAM functionalized $\mathrm{Au}-$ NAA substrate. The effective optical thickness was increased due to continuous binding of CTCs onto anti-EpCAM coated sensor surface. The interferometric signals were changed a bit due to unbound CTCs which were removed from the surface after washing. The detection limit of CTCs was found to be less than 1000 cells/mL, which is significant in practical 


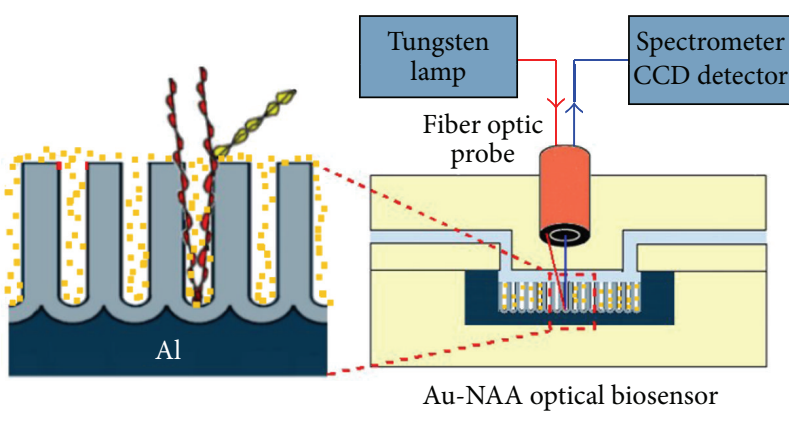

(a)

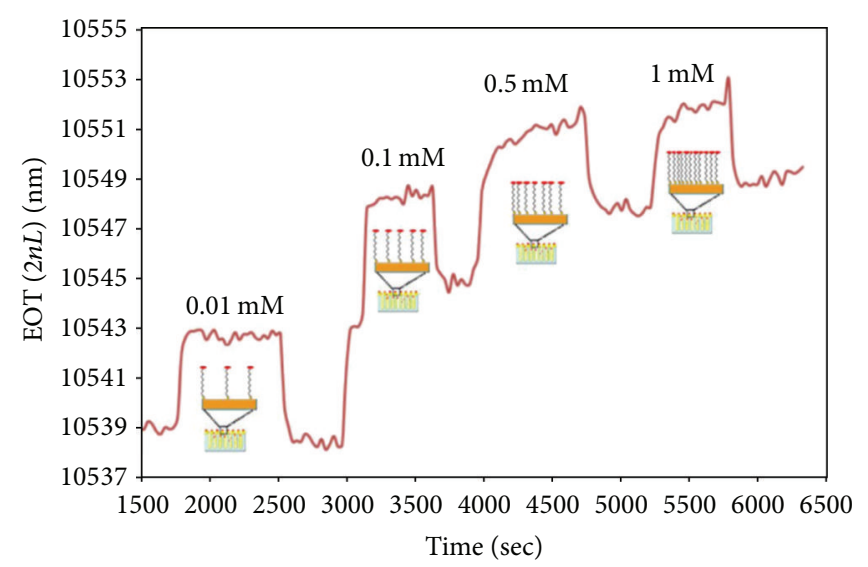

(b)

FIGURE 17: (a) Au-NAA placed in microfluidic chip as a biosensor for the detection of alkyl thiol molecule using reflective interferometric spectroscopy; (b) the real-time measurement of concentration with respect to effective optical thickness of on adsorption molecules on AuNAA [61].

applications and clinical diagnosis. It was also observed that, when the flow rate of CTCs was higher, there was no notable increase in effective optical thickness whereas, when the flow rate was low, effective optical thickness was enhanced by slow ascend and continuing the number of CTCs increment on the sensor surface.

5.7. Optical Properties of Au-NAA. The optical properties of Au-NAA for the application in biosensing by using reflectance spectroscopy have been developed by HernándezEguía et al. [97]. NAA substrates were fabricated with various porosities in the range of 14 to $70 \%$. The expected pore diameters were in the range 38.6 to $90 \mathrm{~nm}$, on which $20 \mathrm{~nm}$ $\mathrm{Au}$ was coated by sputtering method. The prepared $\mathrm{Au}-$ NAA samples were analyzed by reflectance spectroscopy and Fabry-Perot interferences (FI). The interference fringes consist of NAA film and are surrounded by the incident medium of air and the substrates were observed. The FI depends on RI of the material; that is, FI was increased upon increase in RI of Au-NAA substrate, whereas, noncoated NAA samples showed decrease in FI due to less RI compared to Au-NAA. The study on controlled thickness of gold layer was performed to obtain better reflectance spectra in the infra-red region. Thus, by controlling the thickness of the gold layer on NAA surface the better performance of the material can be achieved in terms of sensitivity.

\section{Aluminum Coated NAA (NAA/Al) Biosensor}

6.1. Detection of Protein. Further, the improved label-free optical wave guide biosensor based NAA/Al multilayer film has been developed by Hotta et al. for the detection of BSA through optical wave guide spectroscopy [62]. The wave guide experiment was functioned by measuring the reflection spectra of multilayer thin films using Kretschmann configuration. The multilayer film was fitted with glass prism and xenon lamp was used to irradiate the light onto prism. The incident light was slightly adjusted on prism by polarizer. Then, the reflected light was collected in photonic multichannel analyzer. A polydimethylsiloxane (PDMS) flow cell has been used to flow the analyte solution on multilayer film sensor. The irradiated light was incident on analyte solution and reflected spectra have been used for detection of analyte. The different structures of NAA/Al multilayer films were obtained by varying the substrate thickness. Al films which have been coated on glass substrate with thickness of $180 \mathrm{~nm}$ by thermal evaporation and films were anodized with formation of $34 \%$ porosity, and obtained pore diameter and lengths were of 39 and $220 \mathrm{~nm}$, respectively (film a). Similarly, the same deposition method was used to fabricate Al film of thickness $500 \mathrm{~nm}$ and it was anodized with formation of $45 \%$ porosity to get cylindrical pore structured film of diameter and lengths of at 33 and $670 \mathrm{~nm}$, respectively (film b). For the adsorption of BSA on NAA/Al film structures, the films were rinsed in mixture of prostate specific antigen and BSA solutions. The adsorption of BSA has been analyzed with respect to change in reflectivity on NAA/Al multilayer films. The reflectivity increased due to higher RI by increasing the concentration of BSA on film b compared to film a surface. However, it was found that film b was highly red shifted for the adsorption of BSA due to higher surface area, high porosity, and larger pore length with pore size compared to film a. Thus, the sensitivity was more for multilayer structure of film b over film a. Schematic diagram and working principle of detection of BSA on sensor substrate using optical waveguide spectroscopy and graph showing binding on substrate versus concentration of BSA in the solution phase were shown in Figure 18.

Similarly, Yamaguchi et al. fabricated NAA/Al multilayer thin film on glass substrate for detection of binding interaction of different molecules using optical wave guide senor mechanism [98]. Thus, the sensitivity depends on change in the RI of sensor material where analyte molecules were immobilized on their surfaces. The optical wave guide 


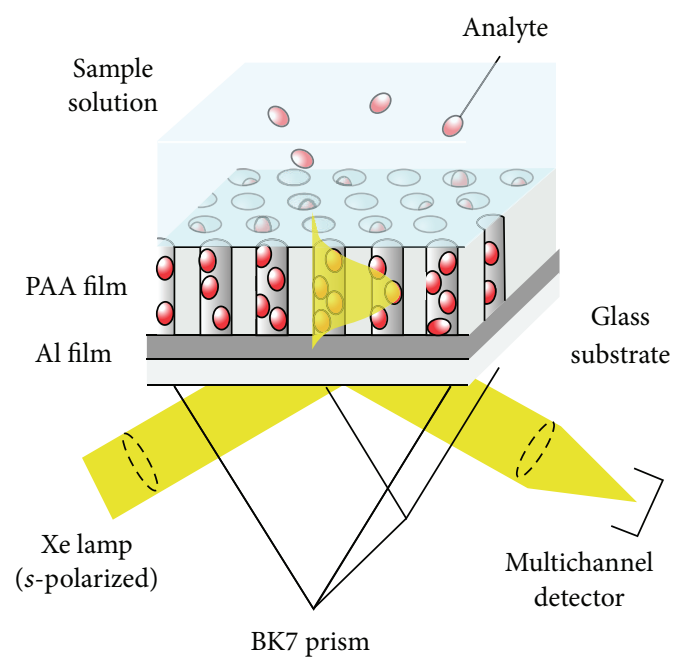

(a)

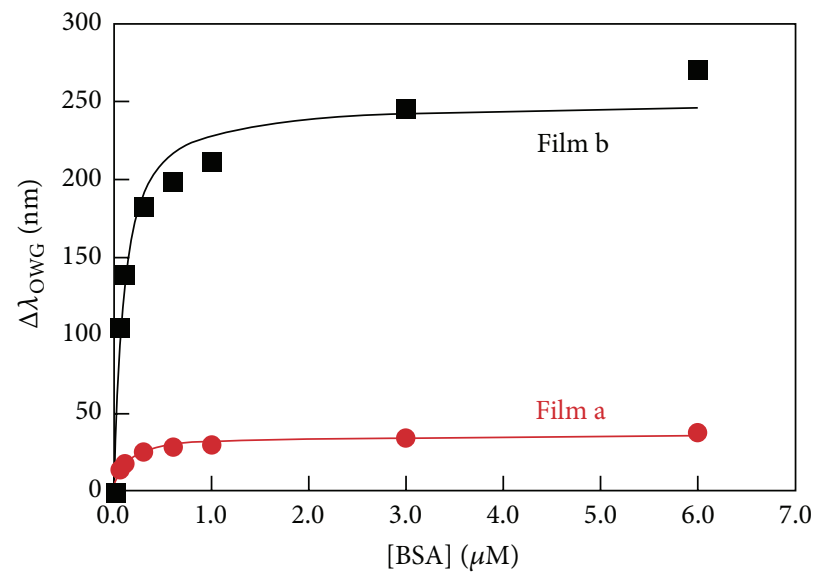

(b)

FIGURE 18: (a) Working principle for the detection of BSA by optical wave guide spectroscopy; (b) shift in the binding on substrate versus the concentration of BSA in the solution phase for film a and film $b$ [62].

sensor got great attention as the small changes in RI of material can be detected; that is, the sensor could detect lower concentration of samples.

\section{Platinum Coated NAA (Pt-NAA) Biosensor}

7.1. Detection of Binding of Prostate Specific Antigen. An et al. developed Pt-NAA biosensor for the improvement of prostate specific antigen binding on sensor surface in interferometric method through pore widening [99]. Earlier same group reported pore widening method on porous $\mathrm{Si}$ where less sensitivity was observed [100]. However, the experiment of Pt-NAA biosensor has improved the sensitivity through fabrication of pore widening structure on NAA. The Pt-NAA was silanized by APTES solution and later this substrate was treated with Prolinker-A solution for binding of biomolecules onto the surface. The Prolinker-A modified sensor surface was bound with prostate specific antigen. The detection of antigen by pore widening on sensor surface by the prostate specific antigen solution of $100 \mathrm{ng} / \mathrm{mL}$ treated with Pt-NAA surface reveals that higher wavelength means rapid detection of binding of antigen molecules on sensor surface. The effective optical thickness has been increased with respect to pore widening on Pt-NAA biosensor surface which is due to larger antigen molecules occupied and bound to ProlinkerA on larger pore and the optical signals were enhanced to get rapid detection through interferometric spectroscopy. Overall, it was found that biosensing of NAA, Au-NAA, Al-NAA, and Pt-NAAs showed better sensitivity towards targets analyzed using different spectroscopy techniques. In NAA, targets were immobilized on their surfaces via binding with antigen-antibody. However, NAA shows less sensitivity compared to other substrates which has been enhanced by shrinking the pore size of $\mathrm{Al}, \mathrm{Au}$, and Pt coated NAA. Further, $\mathrm{Au}-\mathrm{NAA}$ was chosen for biosensing application due to their higher refractive index, surface plasmon, and biocompatibility compared to NAA, Al-NAA, and Pt-NAA. Au-NAA was able to detect biomolecules at very low concentration of $100 \mathrm{ng} / \mathrm{mL}$. In the case of NAA and Al-NAA for the analysis of biomolecules, the concentration was set to be of $0.1 / 1 \mathrm{mg} \mathrm{mL}^{-1}$. The targets were immobilized by covalently binding with antigen-antibody on Au-NAA surface for rapid detection. Lastly, Pt-NAA have better sensitivity compared to Au-NAA but the cost effectiveness of platinum hinders the usage in commercialization purpose.

\section{Conclusion}

In this review, past and recent progress on surface modification and development of NAA platform by the anodization process for suitable biosensing applications have been discussed. By controlling the parameters such as applied voltage, selection of acids, anodization time, and temperature, the precise and highly porous structure of NAA substrates can be fabricated. The methods combined with analytical techniques provide great benefits in terms of technological aspects in biosensors field. Further, the sensitivity and better performance of NAA substrates can be enhanced by coating with gold nanoparticles and also by incorporating with microfluidic devices. However, some process to immobilize biomolecule on substrates requires selected buffer solutions, and some buffer solutions can damage the NAA substrate depending on the $\mathrm{pH}$ level, so the immobilization process may not occur on NAA surfaces which hinders the biosensing process. We believe that in future, the improvement of NAA sensor and modern NAA based sensing platforms will give better results. Further, this review reports sensing mechanism of various kinds of NAA to immobilized biomolecules at lower concentration which was examined by using several spectroscopic techniques. 


\section{Competing Interests}

The authors declare that there is no conflict of interests regarding the publication of this paper.

\section{Acknowledgments}

The authors would like to acknowledge the DST Nanomission (SR/NM/NS-20/2014), India, DST (DST-TM-WTI-2K14213), India, and SERB-DST (YSS/2015/000013), India, for financial support. The authors also would like to thank Jain University, Bangalore, India, for infrastructural support.

\section{References}

[1] I. Sorina, I. Gheorghe, M. Victor, and P. Radu Robert, "Nanostructured zirconia layers as thermal barrier coatings," Incas Bulletin, vol. 3, no. 3, pp. 63-69, 2011.

[2] R. Kumar, O. Al-Dossary, G. Kumar, and A. Umar, "Zinc oxide nanostructures for $\mathrm{NO}_{2}$ gas-sensor applications: a review," Nano-Micro Letters, vol. 7, no. 2, pp. 97-120, 2014.

[3] K. Fan, W. Zhang, T. Peng, J. Chen, and F. Yang, "Application of $\mathrm{TiO}_{2}$ fusiform nanorods for dye-sensitized solar cells with significantly improved efficiency," The Journal of Physical Chemistry C, vol. 115, no. 34, pp. 17213-17219, 2011.

[4] J. Estelrich, E. Escribano, J. Queralt, and M. A. Busquets, "Iron oxide nanoparticles for magnetically-guided and magneticallyresponsive drug delivery," International Journal of Molecular Sciences, vol. 16, no. 4, pp. 8070-8101, 2015.

[5] G. S. Demirer, A. C. Okur, and S. Kizilel, "Synthesis and design of biologically inspired biocompatible iron oxide nanoparticles for biomedical applications," Journal of Materials Chemistry B, vol. 3, no. 40, pp. 7831-7849, 2015.

[6] R. Li, L. Zhang, and P. Wang, "Rational design of nanomaterials for water treatment," Nanoscale, vol. 7, no. 41, pp. 17167-17194, 2015.

[7] S. Ghoshal, A. A. Ansar, S. O. Raja et al., "Superparamagnetic iron oxide nanoparticle attachment on array of micro test tubes and microbeakers formed on p-type silicon substrate for biosensor applications," Nanoscale Research Letters, vol. 6, article 540, 8 pages, 2011.

[8] J. L. Vivero-Escoto and Y.-T. Huang, "Inorganic-organic hybrid nanomaterials for therapeutic and diagnostic imaging applications," International Journal of Molecular Sciences, vol. 12, no. 6, pp. 3888-3927, 2011.

[9] J. W. Kemling, A. J. Qavi, R. C. Bailey, and K. S. Suslick, "Nanostructured substrates for optical sensing," The Journal of Physical Chemistry Letters, vol. 2, no. 22, pp. 2934-2944, 2011.

[10] Y. Liu, P. Kirchesch, T. Graule, A. Liersch, and F. Clemens, "Nanoparticle prepared mechanically stable hierarchically porous silica granulates and their application as oxygen carrier supports for chemical looping combustion," Journal of Materials Chemistry A, vol. 3, no. 22, pp. 11863-11873, 2015.

[11] M. Ge, J. Rong, X. Fang, A. Zhang, Y. Lu, and C. Zhou, "Scalable preparation of porous silicon nanoparticles and their application for lithium-ion battery anodes," Nano Research, vol. 6, no. 3, pp. 174-181, 2013.

[12] L. A. Osminkina, V. A. Sivakov, G. A. Mysov et al., "Nanoparticles prepared from porous silicon nanowires for bio-imaging and sonodynamic therapy," Nanoscale Research Letters, vol. 9, no. 1, article 463, 2014.
[13] F. Keller, M. S. Hunter, and D. L. Robinson, "Structural features of oxide coatings on aluminum," Journal of The Electrochemical Society, vol. 100, no. 9, pp. 411-419, 1953.

[14] A. Thormann, N. Teuscher, M. Pfannmöller, U. Rothe, and A. Heilmann, "Nanoporous aluminum oxide membranes for filtration and biofunctionalization," Small, vol. 3, no. 6, pp.10321040, 2007.

[15] T. Kumeria, M. M. Rahman, A. Santos, J. Ferré-Borrull, L. F. Marsal, and D. Losic, "Structural and optical nanoengineering of nanoporous anodic alumina rugate filters for real-time and label-free biosensing applications," Analytical Chemistry, vol. 86, no. 3, pp. 1837-1844, 2014.

[16] G. Macias, L. P. Hernández-Eguía, J. Ferré-Borrull, J. Pallares, and L. F. Marsal, "Gold-coated ordered nanoporous anodic alumina bilayers for future label-free interferometric biosensors," ACS Applied Materials \& Interfaces, vol. 5, no. 16, pp. 8093-8098, 2013.

[17] K. Noh, K. S. Brammer, C. Choi, S. H. Kim, C. J. Frandsen, and $\mathrm{S}$. Jin, "A new nano-platform for drug release via nanotubular aluminum oxide," Journal of Biomaterials and Nanobiotechnology, vol. 2, no. 3, pp. 226-233, 2011.

[18] C. Jeong, J. Lee, K. Sheppard, and C.-H. Choi, "Air-impregnated nanoporous anodic aluminum oxide layers for enhancing the corrosion resistance of aluminum," Langmuir, vol. 31, no. 40, pp. 11040-11050, 2015.

[19] C.-C. Lu, Y.-S. Huang, J.-W. Huang, C.-K. Chang, and S.-P. Wu, "A macroporous $\mathrm{TiO}_{2}$ oxygen sensor fabricated using anodic aluminium oxide as an etching mask," Sensors, vol. 10, no. 1, pp. 670-683, 2010.

[20] Q. Zhang, J. Xu, F. Fan et al., "Application of porous anodic alumina monolith catalyst in steam reforming of dimethyl ether: $\mathrm{Cu} / \gamma-\mathrm{Al}_{2} \mathrm{O}_{3} / \mathrm{Al}$ catalyst degradation behaviors and catalytic activity improvement by pre-competition impregnation method," Fuel Processing Technology, vol. 119, pp. 52-59, 2014.

[21] N. V. Gaponenko, T. I. Orekhovskaya, I. A. Nikolaenko et al., "Titanium oxide xerogel films in porous aluminum oxide for photocatalytic application," Journal of Applied Spectroscopy, vol. 77, no. 3, pp. 432-436, 2010.

[22] C. S. Law, A. Santos, T. Kumeria, and D. Losic, "Engineered therapeutic-releasing nanoporous anodic alumina-aluminum wires with extended release of therapeutics," ACS Applied Materials and Interfaces, vol. 7, no. 6, pp. 3846-3853, 2015.

[23] L. Wang, X. Qin, D. Ji et al., "Engineering optical properties of metal/porous anodic alumina films for refractometric sensing," Applied Surface Science, vol. 355, pp. 139-144, 2015.

[24] S. D. Kulkarni, K. Choudhari, and C. Santhosh, "Optical interferometric properties of iridescent nanoporous anodic alumina," Procedia Materials Science, vol. 5, pp. 988-994, 2014.

[25] J. Deng and C.-S. Toh, "Impedimetric DNA biosensor based on a nanoporous alumina membrane for the detection of the specific oligonucleotide sequence of dengue virus," Sensors, vol. 13, no. 6, pp. 7774-7785, 2013.

[26] A. R. Walpole, E. P. Briggs, M. Karlsson, E. Pålsgård, and P. R. Wilshaw, "Nano-porous alumina coatings for improved bone implant interfaces," Materialwissenschaft und Werkstofftechnik, vol. 34, no. 12, pp. 1064-1068, 2003.

[27] T. Kumeria, J. Yu, M. Alsawat et al., "Photoswitchable membranes based on peptide-modified nanoporous anodic alumina: toward smart membranes for on-demand molecular transport," Advanced Materials, vol. 27, no. 19, pp. 3019-3024, 2015.

[28] Y. Fan, K. Hotta, A. Yamaguchi et al., "Highly sensitive realtime detection of DNA hybridization by using nanoporous 
waveguide fluorescence spectroscopy," Applied Physics Letters, vol. 105, no. 3, Article ID 031103, 2014.

[29] N. N. Bwana, "Synthesis of highly ordered nanopores on alumina by two-step anodization process," Journal of Nanoparticle Research, vol. 10, no. 2, pp. 313-319, 2008.

[30] H. Masuda and K. Fukuda, "Ordered metal nanohole arrays made by a two-step replication of honeycomb structures of anodic alumina," Science, vol. 268, no. 5216, pp. 1466-1468, 1995.

[31] J.-H. Zhou, J.-P. He, G.-W. Zhao, C.-X. Zhang, J.-S. Zhao, and H.-P. Hu, "Alumina nanostructures prepared by two-step anodization process," Transactions of Nonferrous Metals Society of China, vol. 17, no. 1, pp. 82-86, 2007.

[32] H. Masuda, F. Hasegwa, and S. Ono, "Self-ordering of cell arrangement of anodic porous alumina formed in sulfuric acid solution," Journal of the Electrochemical Society, vol. 144, no. 5, pp. L127-L130, 1997.

[33] H. Masuda, M. Ohya, H. Asoh, M. Nakao, M. Nohtomi, and T. Tamamura, "Photonic crystal using anodic porous alumina," Japanese Journal of Applied Physics, vol. 38, no. 12, pp. L1403L1405, 1999.

[34] W. Lee, R. Ji, U. Gösele, and K. Nielsch, "Fast fabrication of long-range ordered porous alumina membranes by hard anodization," Nature Materials, vol. 5, no. 9, pp. 741-747, 2006.

[35] D. Losic and A. Santos, Nanoporous Alumina: Fabrication, Structure, Properties and Applications, vol. 219 of Springer Series in Material Science, Springer, New York, NY, USA, 2015.

[36] F. Li, L. Zhang, and R. M. Metzger, "On the growth of highly ordered pores in anodized aluminum oxide," Chemistry of Materials, vol. 10, no. 9, pp. 2470-2480, 1998.

[37] W. Lee and S.-J. Park, "Porous anodic aluminum oxide: anodization and templated synthesis of functional nanostructures," Chemical Reviews, vol. 114, no. 15, pp. 7487-7556, 2014.

[38] W. Shi, Y. Shen, D. Ge et al., "Functionalized anodic aluminum oxide (AAO) membranes for affinity protein separation," Journal of Membrane Science, vol. 325, no. 2, pp. 801-808, 2008.

[39] S. Kipke and G. Schmid, "Nanoporous alumina membranes as diffusion controlling systems," Advanced Functional Materials, vol. 14, no. 12, pp. 1184-1188, 2004.

[40] A. Santos, P. Formentín, J. Pallarès, J. Ferré-Borrull, and L. F. Marsal, "Structural engineering of nanoporous anodic alumina funnels with high aspect ratio," Journal of Electroanalytical Chemistry, vol. 655, no. 1, pp. 73-78, 2011.

[41] F. Patel, M. A. Baig, and T. Laoui, "Processing of porous alumina substrate for multilayered ceramic filter," Desalination and Water Treatment, vol. 35, no. 1-3, pp. 33-38, 2011.

[42] Y. Tokudome, K. Nakanishi, K. Kanamori, K. Fujita, H. Akamatsu, and T. Hanada, "Structural characterization of hierarchically porous alumina aerogel and xerogel monoliths," Journal of Colloid and Interface Science, vol. 338, no. 2, pp. 506-513, 2009.

[43] J. Martín, M. Martín-González, J. Francisco Fernández, and O. Caballero-Calero, "Ordered three-dimensional interconnected nanoarchitectures in anodic porous alumina," Nature Communications, vol. 5, article 5130, 2014.

[44] A. Santos, T. Kumeria, Y. Wang, and D. Losic, "In situ monitored engineering of inverted nanoporous anodic alumina funnels: on the precise generation of 3D optical nanostructures," Nanoscale, vol. 6, no. 17, pp. 9991-9999, 2014.

[45] Y. C. Sui, B. Z. Cui, R. Guardián, D. R. Acosta, L. Martínez, and R. Perez, "Growth of carbon nanotubes and nanofibres in porous anodic alumina film," Carbon, vol. 40, no. 7, pp. 10111016, 2002.
[46] S. Wang, G. J. Yu, J. L. Gong, D. Z. Zhu, and H. H. Xia, "Largearea uniform nanodot arrays embedded in porous anodic alumina," Nanotechnology, vol. 18, no. 1, Article ID 015303, 2007.

[47] G. E. J. Poinern, X. T. Le, M. Hager, T. Becker, and D. Fawcett, "Electrochemical synthesis, characterisation, and preliminary biological evaluation of an anodic aluminium oxide membrane with a pore size of 100 nanometres for a potential cell culture substrate," American Journal of Biomedical Engineering, vol. 3, no. 6, pp. 119-131, 2013.

[48] N. Verma, K. C. Singh, and J. Jindal, "Fabrication of nanomaterials on porous anodic alumina template using various techniques," Indian Journal of Advances in Chemical Science, vol. 3, no. 3, pp. 235-246, 2015.

[49] K. Kant, J. Yu, C. Priest, J. G. Shapter, and D. Losic, "Impedance nanopore biosensor: influence of pore dimensions on biosensing performance," Analyst, vol. 139, no. 5, pp. 1134-1140, 2014.

[50] S. D. Alvarez, C.-P. Li, C. E. Chiang, I. K. Schuller, and M. J. Sailor, "A label-free porous alumina interferometric immunosensor," ACS Nano, vol. 3, no. 10, pp. 3301-3307, 2009.

[51] K. Hotta, A. Yamaguchi, and N. Teramae, "Deposition of polyelectrolyte multilayer film on a nanoporous alumina membrane for stable label-free optical biosensing," The Journal of Physical Chemistry C, vol. 116, no. 44, pp. 23533-23539, 2012.

[52] Y. Chen, A. Santos, Y. Wang et al., "Biomimetic nanoporous anodic alumina distributed bragg reflectors in the form of films and microsized particles for sensing applications," ACS Applied Materials and Interfaces, vol. 7, no. 35, pp. 19816-19824, 2015.

[53] F. S. H. Krismastuti, H. Bayat, N. H. Voelcker, and H. Schönherr, "Real time monitoring of layer-by-layer polyelectrolyte deposition and bacterial enzyme detection in nanoporous anodized aluminum oxide," Analytical Chemistry, vol. 87, no. 7, pp. 38563863, 2015.

[54] A. Santos, G. Macías, J. Ferré-Borrull, J. Pallarès, and L. F. Marsal, "Photoluminescent enzymatic sensor based on nanoporous anodic alumina," ACS Applied Materials \& Interfaces, vol. 4, no. 7, pp. 3584-3588, 2012.

[55] A. Dhathathreyan, "Real-time monitoring of invertase activity immobilized in aluminum oxide-nanoporous," The Journal of Physical Chemistry B, vol. 115, no. 20, pp. 6678-6682, 2011.

[56] M. Nemati, A. Santos, T. Kumeria, and D. Losic, "Labelfree real-time quantification of enzyme levels by interferometric spectroscopy combined with gelatin-modified nanoporous anodic alumina photonic films," Analytical Chemistry, vol. 87, no. 17, pp. 9016-9024, 2015.

[57] S.-H. Yeom, O.-G. Kim, B.-H. Kang et al., "Highly sensitive nano-porous lattice biosensor based on localized surface plasmon resonance and interference," Optics Express, vol. 19, no. 23, pp. 22882-22891, 2011.

[58] H. M. Hiep, H. Yoshikawa, and E. Tamiya, "Interference localized surface plasmon resonance nanosensor tailored for the detection of specific biomolecular interactions," Analytical Chemistry, vol. 82, no. 4, pp. 1221-1227, 2010.

[59] D.-K. Kim, K. Kerman, M. Saito et al., "Label-free DNA biosensor based on localized surface plasmon resonance coupled with interferometry," Analytical Chemistry, vol. 79, no. 5, pp. 1855$1864,2007$.

[60] G. Das, N. Patra, A. Gopalakrishnan et al., "Fabrication of large-area ordered and reproducible nanostructures for SERS biosensor application," Analyst, vol. 137, no. 8, pp. 1785-1792, 2012. 
[61] T. Kumeria and D. Losic, "Controlling interferometric properties of nanoporous anodic aluminium oxide," Nanoscale Research Letters, vol. 7, article 88, 2012.

[62] K. Hotta, A. Yamaguchi, and N. Teramae, "Nanoporous waveguide sensor with optimized nanoarchitectures for highly sensitive label-free biosensing," ACS Nano, vol. 6, no. 2, pp. 1541-1547, 2012.

[63] A. Yin, M. Tzolov, D. Cardimona, L. Guo, and J. Xu, "Fabrication of highly ordered anodic aluminium oxide templates on silicon substrates," IET Circuits, Devices and Systems, vol. 1, no. 3, pp. 205-209, 2007.

[64] D. Lo and R. A. Budiman, "Fabrication and characterization of porous anodic alumina films from impure aluminum foils," Journal of the Electrochemical Society, vol. 154, no. 1, pp. C60C66, 2007.

[65] T. Nagaura, F. Takeuchi, and S. Inoue, "Fabrication and structural control of anodic alumina films with inverted cone porous structure using multi-step anodizing," Electrochimica Acta, vol. 53, no. 5, pp. 2109-2114, 2008.

[66] T. Hussain, A. T. Shah, K. Shehzad et al., "Formation of self-ordered porous anodized alumina template for growing tungsten trioxide nanowires," International Nano Letters, vol. 5, no. 1, pp. 37-41, 2015.

[67] H.-C. Na, T.-J. Sung, S.-H. Yoon et al., "Formation of unidirectional nanoporous structures in thickly anodized aluminum oxide layer," Transactions of Nonferrous Metals Society of China, vol. 19, no. 4, pp. 1013-1017, 2009.

[68] H. Tsuchiya, S. Berger, J. M. Macak, A. G. Muñoz, and P. Schmuki, "A new route for the formation of self-organized anodic porous alumina in neutral electrolytes," Electrochemistry Communications, vol. 9, no. 4, pp. 545-550, 2007.

[69] H. Asoh, K. Nishio, M. Nakao, A. Yokoo, T. Tamamura, and H. Masuda, "Fabrication of ideally ordered anodic porous alumina with $63 \mathrm{~nm}$ hole periodicity using sulfuric acid," Journal of Vacuum Science \& Technology B, vol. 19, no. 2, pp. 569-572, 2001.

[70] F. Zhang, X. Liu, C. Pan, and J. Zhu, "Nano-porous anodic aluminium oxide membranes with $6-19 \mathrm{~nm}$ pore diameters formed by a low-potential anodizing process," Nanotechnology, vol. 18, no. 34, Article ID 345302, 2007.

[71] Y. Wang, A. Santos, A. Evdokiou, and D. Losic, "Rational design of ultra-short anodic alumina nanotubes by short-time pulse anodization," Electrochimica Acta, vol. 154, pp. 379-386, 2015.

[72] A. L. Friedman, D. Brittain, and L. Menon, "Roles of $p H$ and acid type in the anodic growth of porous alumina," The Journal of Chemical Physics, vol. 127, no. 15, Article ID 154717, 2007.

[73] J. Ferré-Borrull, J. Pallarès, G. Macías, and L. F. Marsal, "Nanostructural engineering of nanoporous anodic alumina for biosensing applications," Materials, vol. 7, no. 7, pp. 5225-5253, 2014.

[74] T. Kumeria, A. Santos, and D. Losic, "Nanoporous anodic alumina platforms: engineered surface chemistry and structure for optical sensing applications," Sensors, vol. 14, no. 7, pp. 1187811918, 2014

[75] A. Santos, T. Kumeria, and D. Losic, "Nanoporous anodic aluminum oxide for chemical sensing and biosensors," TrACTrends in Analytical Chemistry, vol. 44, pp. 25-38, 2013.

[76] A. Santos, T. Kumeria, and D. Losic, "Nanoporous anodic alumina: a versatile platform for optical biosensors," Materials, vol. 7, no. 6, pp. 4297-4320, 2014.

[77] A. Santos, V. S. Balderrama, M. Alba et al., "Nanoporous anodic alumina barcodes: toward smart optical biosensors," Advanced Materials, vol. 24, no. 8, pp. 1050-1054, 2012.
[78] A. Santos, V. S. Balderrama, M. Alba et al., “Tunable fabry-pérot interferometer based on nanoporous anodic alumina for optical biosensing purposes," Nanoscale Research Letters, vol. 7, no. 1, article 370, pp. 1-10, 2012.

[79] A. Santos, T. Kumeria, and D. Losic, "Optically optimized photoluminescent and interferometric biosensors based on nanoporous anodic alumina: a comparison," Analytical Chemistry, vol. 85, no. 16, pp. 7904-7911, 2013.

[80] P. Takmakov, I. Vlassiouk, and S. Smirnov, "Application of anodized aluminum in fluorescence detection of biological species," Analytical and Bioanalytical Chemistry, vol. 385, no. 5, pp. 954-958, 2006.

[81] I. Vlassiouk, A. Krasnoslobodtsev, S. Smirnov, and M. Germann, "Direct' detection and separation of DNA using nanoporous alumina filters," Langmuir, vol. 20, no. 23, pp. 99139915, 2004.

[82] C. Fu, Y. Gu, Z. Wu, Y. Wang, S. Xu, and W. Xu, "Surfaceenhanced Raman scattering (SERS) biosensing based on nanoporous dielectric waveguide resonance," Sensors and Actuators B: Chemical, vol. 201, pp. 173-176, 2014.

[83] K. H. A. Lau, L.-S. Tan, K. Tamada, M. S. Sander, and W. Knoll, "Highly sensitive detection of processes occurring inside nanoporous anodic alumina templates: a waveguide optical study," Journal of Physical Chemistry B, vol. 108, no. 30, pp. 10812-10818, 2004.

[84] S. Pan and L. J. Rothberg, "Interferometric sensing of biomolecular binding using nanoporous aluminum oxide templates," Nano Letters, vol. 3, no. 6, pp. 811-814, 2003.

[85] P. Takmakov, I. Vlassiouk, and S. Smirnov, "Hydrothermally shrunk alumina nanopores and their application to DNA sensing," Analyst, vol. 131, no. 11, pp. 1248-1253, 2006.

[86] M. P. Nicholas, L. Rao, and A. Gennerich, "Covalent immobilization of microtubules on glass surfaces for molecular motor force measurements and other single-molecule assays," Methods in Molecular Biology, vol. 1136, pp. 137-169, 2014.

[87] J.-M. Moon, D. Akin, Y. Xuan, P. D. Ye, P. Guo, and R. Bashir, "Capture and alignment of phi29 viral particles in sub-40 nanometer porous alumina membranes," Biomedical Microdevices, vol. 11, no. 1, pp. 135-142, 2009.

[88] M. Darder, P. Aranda, M. Hernández-Vélez, E. Manova, and E. Ruiz-Hitzky, "Encapsulation of enzymes in alumina membranes of controlled pore size," Thin Solid Films, vol. 495, no. 1-2, pp. 321-326, 2006.

[89] A. Hierro-Rodriguez, P. Rocha-Rodrigues, F. Valdés-Bango et al., "On the anodic aluminium oxide refractive index of nanoporous templates," Journal of Physics D: Applied Physics, vol. 48, no. 45, Article ID 455105, 2015.

[90] D.-K. Kim, K. Kerman, H. M. Hiep et al., "Label-free optical detection of aptamer-protein interactions using gold-capped oxide nanostructures," Analytical Biochemistry, vol. 379, no. 1, pp. 1-7, 2008.

[91] C. Toccafondi, R. La Rocca, A. Scarpellini, M. Salerno, G. Das, and S. Dante, "Thin nanoporous alumina-based SERS platform for single cell sensing," Applied Surface Science, vol. 351, pp. 738$745,2015$.

[92] C. Toccafondi, S. Thorat, R. La Rocca et al., "Multifunctional substrates of thin porous alumina for cell biosensors," Journal of Materials Science: Materials in Medicine, vol. 25, no. 10, pp. 2411-2420, 2014.

[93] T. Kumeria, M. D. Kurkuri, K. R. Diener, L. Parkinson, and D. Losic, "Label-free reflectometric interference microchip 
biosensor based on nanoporous alumina for detection of circulating tumour cells," Biosensors and Bioelectronics, vol. 35, no. 1, pp. 167-173, 2012.

[94] M. D. Kurkuri, F. Al-Ejeh, J. Y. Shi et al., "Plasma functionalized PDMS microfluidic chips: towards point-of-care capture of circulating tumor cells," Journal of Materials Chemistry, vol. 21, no. 24, pp. 8841-8848, 2011.

[95] P. Patil, Madhuprasad, T. Kumeria, D. Losic, and M. Kurkuri, "Isolation of circulating tumour cells by physical means in a microfluidic device: a review," RSC Advances, vol. 5, no. 109, pp. 89745-89762, 2015.

[96] B. Thierry, M. Kurkuri, J. Y. Shi, L. E. M. P. Lwin, and D. Palms, "Herceptin functionalized microfluidic polydimethylsiloxane devices for the capture of human epidermal growth factor receptor 2 positive circulating breast cancer cells," Biomicrofluidics, vol. 4, no. 3, Article ID 032205, 2010.

[97] L. P. Hernández-Eguía, J. Ferré-Borrull, G. Macias, J. Pallarès, and L. F. Marsal, "Engineering optical properties of goldcoated nanoporous anodic alumina for biosensing," Nanoscale Research Letters, vol. 9, no. 1, article 414, 2014.

[98] A. Yamaguchi, K. Hotta, and N. Teramae, "Optical waveguide sensor based on a porous anodic alumina/aluminum multilayer film," Analytical Chemistry, vol. 81, no. 1, pp. 105-111, 2009.

[99] H. C. An, J. Y. An, and B.-W. Kim, "Improvement of sensitivity in an interferometry by controlling pore size on the anodic aluminum oxide chip pore-widening technique," Korean Journal of Chemical Engineering, vol. 26, no. 1, pp. 160-164, 2009.

[100] A. Janshoff, K.-P. S. Dancil, C. Steinem et al., "Macroporous p-type silicon fabry-perot layers. Fabrication, characterization, and applications in biosensing," Journal of the American Chemical Society, vol. 120, no. 46, pp. 12108-12116, 1998. 

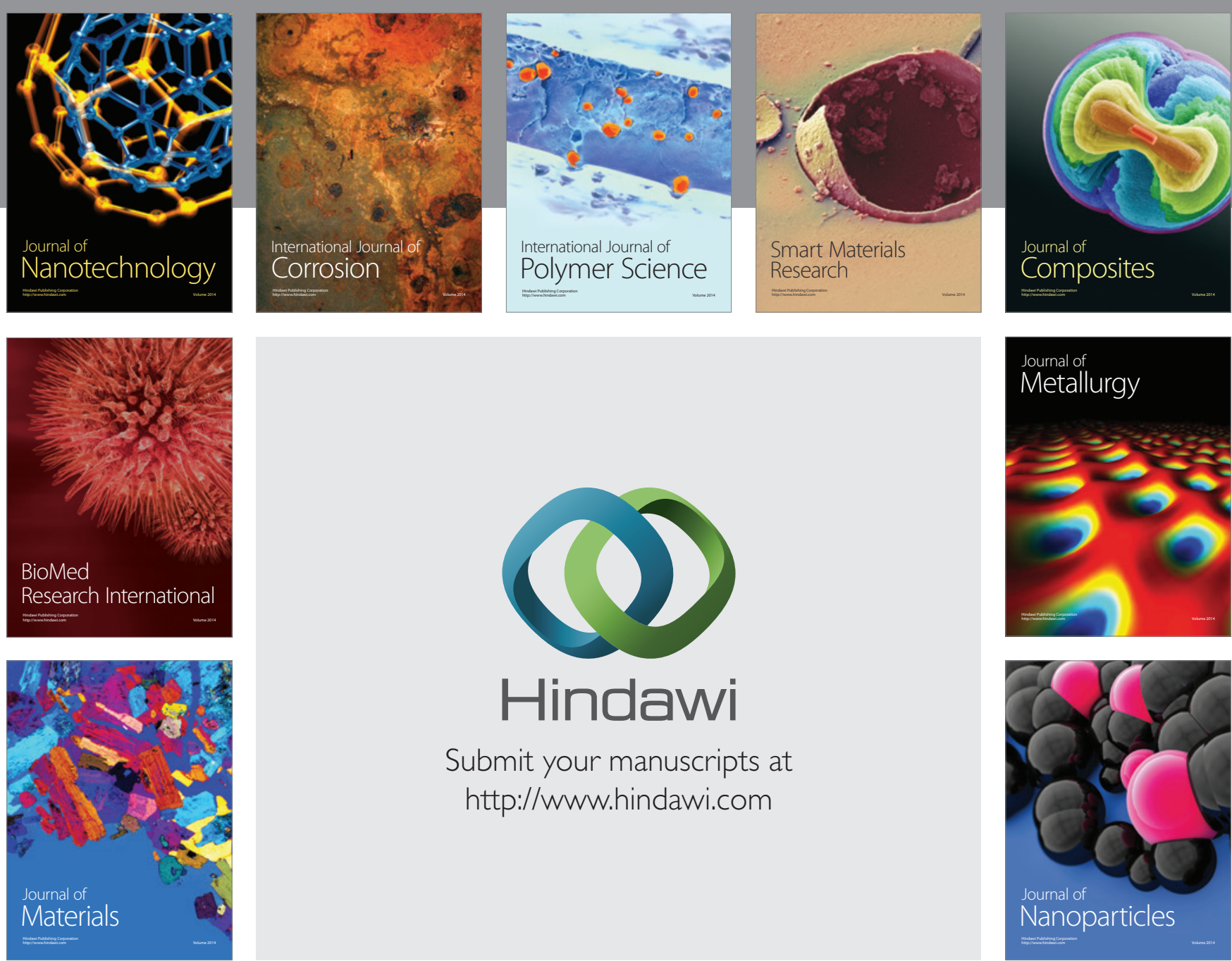

\section{Hindawi}

Submit your manuscripts at

http://www.hindawi.com

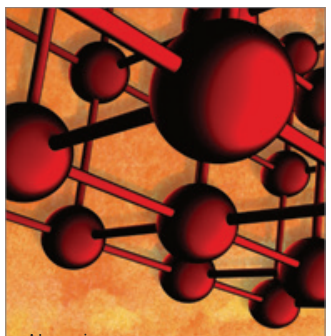

Materials Science and Engineering
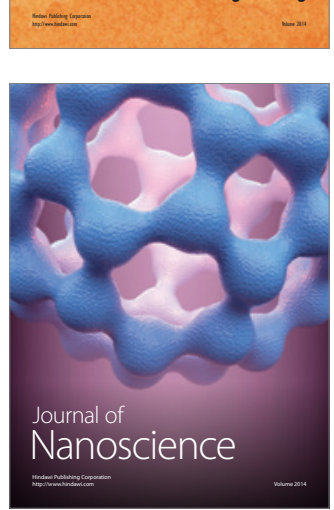
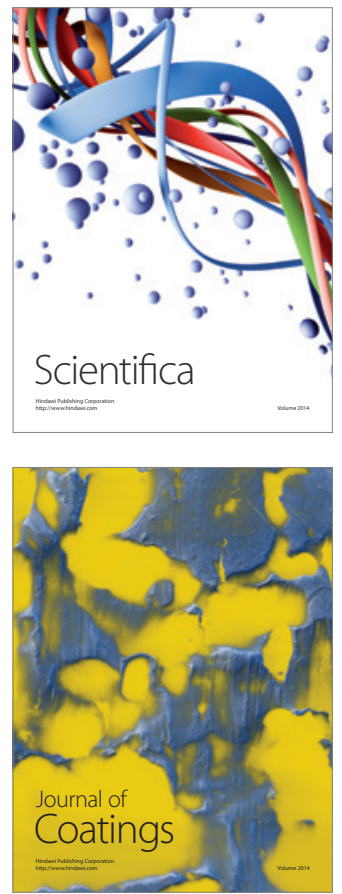
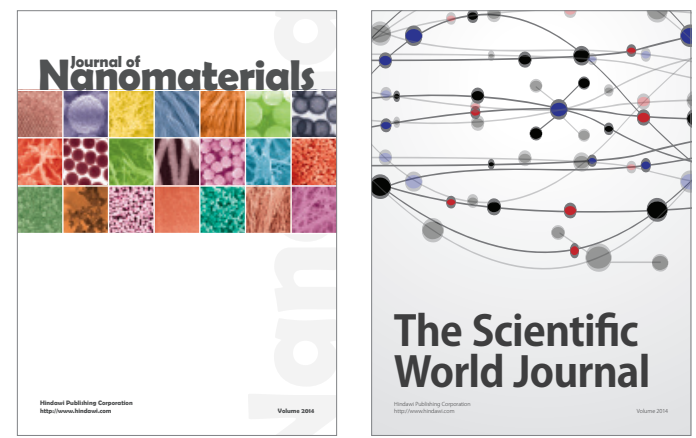

The Scientific World Journal
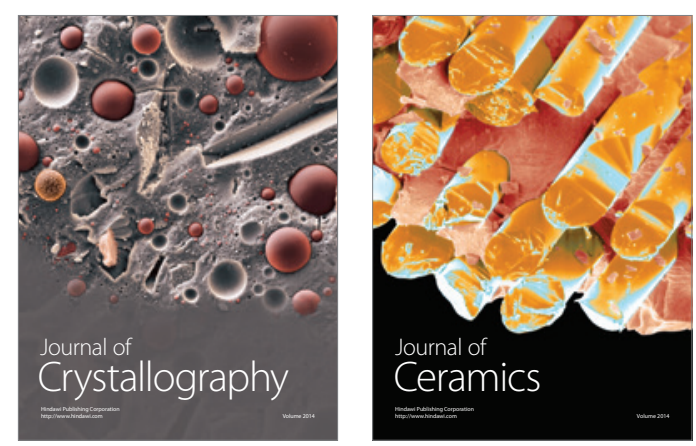
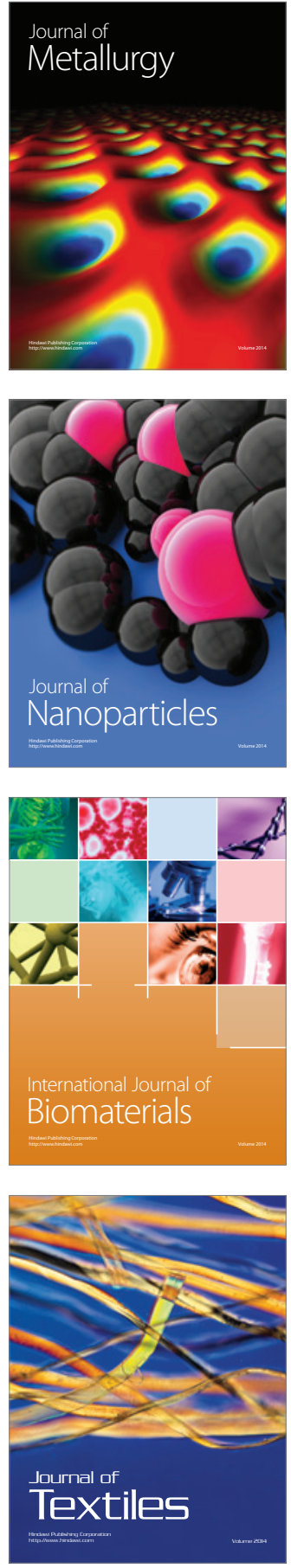